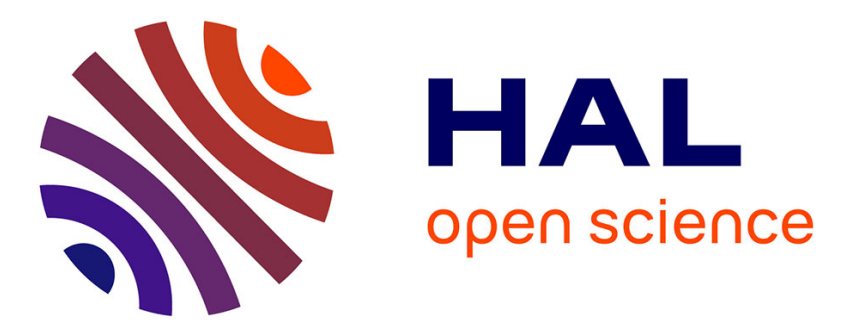

\title{
Non-intrusive reduced-order modeling of parameterized electromagnetic scattering problems using cubic spline interpolation
}

Kun Li, Ting-Zhu Huang, Liang Li, Stéphane Lanteri

\section{- To cite this version:}

Kun Li, Ting-Zhu Huang, Liang Li, Stéphane Lanteri. Non-intrusive reduced-order modeling of parameterized electromagnetic scattering problems using cubic spline interpolation. Journal of Scientific Computing, 2021, 87 (2), 10.1007/s10915-021-01467-2 . hal-03437698

\author{
HAL Id: hal-03437698 \\ https://hal.inria.fr/hal-03437698
}

Submitted on 22 Nov 2021

HAL is a multi-disciplinary open access archive for the deposit and dissemination of scientific research documents, whether they are published or not. The documents may come from teaching and research institutions in France or abroad, or from public or private research centers.
L'archive ouverte pluridisciplinaire HAL, est destinée au dépôt et à la diffusion de documents scientifiques de niveau recherche, publiés ou non, émanant des établissements d'enseignement et de recherche français ou étrangers, des laboratoires publics ou privés. 


\title{
Non-intrusive reduced-order modeling of parameterized electromagnetic scattering problems using cubic spline interpolation
}

\author{
Kun $\mathrm{Li}^{\mathrm{a}}$, Ting-Zhu Huang ${ }^{\mathrm{b}, *}$, Liang $\mathrm{Li}^{\mathrm{b}, *}$, Stéphane Lanteri ${ }^{\mathrm{c}}$ \\ ${ }^{a}$ School of Economic Mathematics, Southwestern University of Finance and Economics, 611130, Chengdu, P.R. \\ China \\ ${ }^{b}$ School of Mathematical Sciences, University of Electronic Science and Technology of China, 611731, Chengdu, \\ P.R. China \\ ${ }^{c}$ INRIA, 2004 Route des Lucioles, BP 9306902 Sophia Antipolis Cedex, France
}

\begin{abstract}
This paper presents a non-intrusive model order reduction (MOR) for the solution of parameterized electromagnetic scattering problems, which needs to prepare a database offline of full-order solution samples (snapshots) at some different parameter locations. The snapshot vectors are produced by a high order discontinuous Galerkin time-domain (DGTD) solver formulated on an unstructured simplicial mesh. Because the second dimension of snapshots matrix is large, a two-step or nested proper orthogonal decomposition (POD) method is employed to extract timeand parameter-independent POD basis functions. By using the singular value decomposition (SVD) method, the principal components of the projection coefficient matrices (also referred to as the reduced coefficient matrices) of full-order solutions onto the RB subspace are extracted. A cubic spline interpolation-based (CSI) approach is proposed to approximate the dominating time- and parameter-modes of the reduced coefficient matrices without resorting to Galerkin projection. The generation of snapshot vectors, the construction of POD basis functions and the approximation of reduced coefficient matrices based on the CSI method are completed during the offline stage. The RB solutions for new time and parameter values can be rapidly recovered via outputs from the interpolation models in the online stage. In particular, the offline and online stages of the proposed RB method, termed as the POD-CSI method, are completely decoupled, which ensures the computational validity of the method. Moreover, a surrogate error model is constructed as an efficient error estimator for the POD-CSI method. Numerical experiments for the scattering of plane wave by a 2-D dielectric cylinder and a multi-layer heterogeneous medium nicely illustrate the performance of POD-CSI method.
\end{abstract}

Keywords: Non-intrusive reduced-order modeling, reduced basis method, proper orthogonal decomposition, cubic spline interpolation, parameterized electromagnetic scattering problems, discontinuous Galerkin method

\section{Introduction}

A variety of physical systems occurring in nature and in engineering are usually described by partial differential equations (PDEs) whose numerical solution requires considerable computational effort, especially in the case of parameterized PDEs [1], which often need to be solved for a

\footnotetext{
${ }^{*}$ Corresponding author

Email addresses: rclikun@163.com, likun@swufe.edu.cn (Kun Li), tzhuang@uestc.edu.cn (Ting-Zhu Huang), plum_liliang@uestc.edu.cn, plum.liliang@gmail.com (Liang Li), Stephane.Lanteri@inria.fr (Stéphane Lanteri)
} 
range of parameter values $[2,3]$. Such a situation is for instance encountered when studying electromagnetic wave propagation problems in complex geometries for different input parameters including frequency, direction of the incident wave, geometric dimensions, as well as material properties [4], which are described by the system of time-domain Maxwell's equations. The most widely used method for solving this system is the finite difference time-domain (FDTD) method introduced by Yee [5] in 1966. The FDTD method is easy to implement and computationally efficient for many problems involving propagation domains or scattering structures with planar geometries. Besides, more flexible methods formulated on unstructured meshes or possibly non-conforming meshes have also been devised to deal with curved geometries. In particular, the discontinuous Galerkin time-domain (DGTD) method, which can be regarded as a combination of the finite element (FE) and finite volume (FV) methods [6], has progressively emerged as a viable alternative to the well established FDTD method. The DGTD method has several attractive features, such as easy adaptation to complex geometries and material composition, local approximation order strategy, as well as easy parallelization, and does not require the inversion of global mass matrix when combined with a fully explicit time scheme.

Although the DGTD method is highly accurate and widely applicable, it forms a high dimensional model (HDM) due to the duplication of the degrees of freedom (DoF) on the boundaries of the elements, so it is often expensive in terms of both CPU time and memory demands for computing high fidelity solutions, especially in the context of multi-entry analysis including optimal design and uncertainty quantification (UQ), where it is often required to repeatedly solve the HDM over a large number of parameter values. Hence, it is extremely important to develop a reduced-order model (ROM), which allows for rapidly, inexpensively, and effectively providing the numerical solution of the problem for a range of parameter values while maintaining a sufficiently high accuracy. The reduced basis (RB) method based on a offline-online stages implementation is a widely used model order reduction (MOR) technique $[1,7,8]$. During the offline stage, the time- and parameter-independent RB functions are extracted from the collection of full-order solutions (snapshots) generated by the DGTD method at some different parameter locations. There are popular methods comprising the error estimator/indicatorbased greedy algorithm $[9,10,11]$ and the singular value decomposition (SVD)-based proper orthogonal decomposition (POD) method $[4,12,13,14,15,16]$ to generate the RB functions. In particular, the greedy approach is not feasible without a natural criteria for the generation of RB functions [17, 18]. Here a two-step or nested POD method is applied because the second dimension of snapshots matrix is large, where the POD method is implemented separately for time and parameter spaces.

In the RB method, the reduced-order solution for a new time/parameter value can be understood as the projection of the high fidelity solution onto the reduced subspace spanned by the RB functions [18], in which the projection coefficients, also called the reduced-order coefficients, need to be calculated in the online stage. According to the method of computing the reduced-order coefficients, the $\mathrm{RB}$ methods can be classified into two categories $[2,18]$ : intrusive and non-intrusive. In the former, the ROM is firstly established by using Galerkin projection $[4,19,20,21,22]$ or other means $[23,24]$, which yields a finite-dimensional dynamical system with the smallest possible $\operatorname{DoF}[16,25,26]$, and then the reduced-order coefficients are calculated by solving the ROM with the time scheme (e.g., the second order leap-frog $\left(\mathrm{LF}_{2}\right)$ scheme). MOR for FDTD [27, 28], DGTD [16, 26], and hybridizable discontinuous Galerkin (HDG) $[4,29]$ methods based on the projection technique has proved to be a powerful method to save computational cost for the PDEs. However, one of the major drawbacks of the intrusive methods is that they require access to the HDM [30]. Moreover, the projection-based RB methods can provide limited computational gain especially for complex nonlinear problems and suffer from instabilities [18]. The non-intrusive MOR, which aims to construct approximate mappings 
from given training dataset [31], has recently attracted great interest in the field of scientific computing, and is currently developing rapidly with the emergence of various viewpoints [32]. The reduced-order coefficients for a new time/parameter value then can be obtained via the approximate mappings $[18,33]$. The high fidelity solver is only used to generate the snapshots and the training dataset, guaranteeing a complete decoupling between the online evaluation and the offline training. There is a lot of work on the non-intrusive method in the past, such as the regression-based non-intrusive RB methods including the tensor decomposition based regression (see, e.g., for parametrized time-dependent problem [2]), the artificial neural networks (ANNs) based regression (see, e.g., for steady-state problem [34], for combustion problem [18], and for transient flow [35]), and the Gaussian processes regression (GPR) based regression (see, e.g., for nonlinear structural analysis [36], and for compressible flow [31]), and the interpolation-based non-intrusive RB methods comprising the radial basis function (RBF) interpolations (see, e.g., for parametrized time-dependent PDE [37], for multiphase flows in porous media [38], and for shallow water equations [39]), the polynomial interpolations (see, e.g., for parametrized timedependent problems [40], for stochastic representations in UQ analysis [41]), and the cubic spline interpolations (CSI) (see, e.g., for parametric applications in transonic aerodynamics [42], and for non-linear parametrized physical problems [43]).

In this paper, to ensure the reliability and effectiveness of non-intrusive RB method, we use the SVD method to extract the principal components of the training dataset, and then use the data-driven (regression or interpolation) method to approximate the dominating discrete timeand parameter-modes, where hundreds of independent regression or interpolation experiments need to be completed in the parameterized time-domain electromagnetic scattering problems. The GPR and ANNs methods are widely used, especially the GPR method is still effective for problems with small data set with proper chosen initialization and kernel, but its initial values may be completely different for hundreds of discrete time- and parameter-modes, and they can not loop directly, and only adjust manually. In addtion, in the training process of GPRs, the optimization of hyperparameters is time-consuming if the library functions of MATLAB is directly used. The spline interpolation is a data-driven method commonly used to obtain smooth curves in industrial design, among which the CSI method is one of the most widely used. Hence, the non-intrusive RB method based on the POD and CSI techniques, termed as the POD-CSI method, is developed for solving parameterized electromagnetic scattering problems in this study, in which the CSI method is used to approximate the mappings between the time/parameter valuers and the time/parameter-modes of reduced-order coefficient matrices. In addition, the projection error of the snapshot vectors onto the RB subspace construced by the two-step POD method is presented via Schmidt-Eckart-Young (S-E-Y) theorem. After obtaining the ROM, the error of POD-CSI method based on the projection error at all training points is given. Moreover, an error surrogate model is constructed to estimate the error of the POD-CSI method at some testing points.

The remainder of this paper is organised as follows. The time-domain Maxwell's equations and the corresponding discontinuous Galerkin (DG) scheme are briefly introduced in Section 2. The non-intrusive ROM is presented in Section 3. Section 4 briefly introduces the CSI method and the structure of the CSI-based interpolation of reduced-order coefficient matrices. Section 5 presents the numerical results including the scattering of plane wave by a 2-D a dielectric cylinder and a multi-layer heterogeneous medium. The conclusions are drawn in Section 6 .

\section{Mathematical modeling}

This work is concerned with the scenario where a scatterer $\Omega_{s}$ lying in a dielectric medium $\Omega \subset \mathcal{R}^{3}$ (e.g. vacuum) is illuminated by an incident wave, which is governed by the system of 
time-domain Maxwell's equations (normalized form)

$$
\left\{\begin{array}{l}
\mu_{r} \frac{\partial \mathbf{H}(\mathbf{x}, t)}{\partial t}+\nabla \times \mathbf{E}(\mathbf{x}, t)=0, \\
\varepsilon_{r} \frac{\partial \mathbf{E}(\mathbf{x}, t)}{\partial t}-\nabla \times \mathbf{H}(\mathbf{x}, t)=0,
\end{array} \quad \forall(\mathbf{x}, t) \in \Omega \times \mathcal{T},\right.
$$

where $\mathbf{E}=\left(E_{x}, E_{y}, E_{z}\right)^{T}$ and $\mathbf{H}=\left(H_{x}, H_{y}, H_{z}\right)^{T}$ respectively denote the electric and magnetic fields (the symbol ${ }^{T}$ denotes the transposition); $\mathcal{T} \subset \mathcal{R}^{+}$is the time domain; $\varepsilon_{r}$ and $\mu_{r}$ are the relative permittivity and permeability, respectively. In particular, we consider $\theta=$ $\left(\varepsilon_{r}^{(1)}, \varepsilon_{r}^{(2)}, \cdots, \varepsilon_{r}^{(d)}\right) \in \mathcal{P} \subset \mathcal{R}^{d}$ as the problem's parameters with $\varepsilon_{r}^{(i)}(i=1,2, \cdots, d)$ being the relative permittivity in the $i$-th domain of $\Omega_{s}, \mathcal{P}$ being the parameter domain, and $d$ being the number of parameters. Our goal is to solve the system (1) in $\Omega$ with boundary $\partial \Omega=\Gamma_{a}$, where we impose the first-order Silver-Müller absorbing boundary condition (ABC)

$$
\mathbf{n} \times \mathbf{E}+Z \mathbf{n} \times(\mathbf{n} \times \mathbf{H})=\mathbf{n} \times \mathbf{E}^{\text {inc }}+Z \mathbf{n} \times\left(\mathbf{n} \times \mathbf{H}^{\text {inc }}\right) .
$$

Here, $\mathbf{n}$ denotes the unit normal vector pointing outward to $\partial \Omega, \mathbf{E}^{\text {inc }}$ and $\mathbf{H}^{\text {inc }}$ stand for the incident fields, and $Z=\sqrt{\mu_{r} / \varepsilon_{r}}$. System (1) with the boundary condition is completed with initial conditions $\mathbf{E}(\mathbf{x}, 0)=\mathbf{E}_{0}(\mathbf{x})$, and $\mathbf{H}(\mathbf{x}, 0)=\mathbf{H}_{0}(\mathbf{x})$ for $\mathbf{x} \in \Omega \subset \mathcal{R}^{3}$, where $\mathbf{E}_{0}$ and $\mathbf{H}_{0}$ denote some given functions.

In a discontinuous Galerkin (DG) framework the computational domain $\Omega$ is divided into elements $\Omega_{\hbar}=\bigcup_{i=1}^{\mathcal{N}_{\Omega}} K_{i}$ with $\mathcal{N}_{\Omega}$ being the number of elements. Then the DG method seeks an approximate solution $\left(\mathbf{E}_{h}, \mathbf{H}_{h}\right)$ in the space $\mathbf{V}_{h} \times \mathbf{V}_{h}$ that satisfies for all $K_{i}$ in $\Omega_{\hbar}$

$$
\begin{cases}\left(\mu_{r} \frac{\partial \mathbf{H}_{h}}{\partial t}, \mathbf{v}\right)_{K_{i}}+\left(\mathbf{E}_{h}, \text { curlv }\right)_{K_{i}}-\left\langle\mathbf{E}_{h}^{*} \times \mathbf{n}, \mathbf{v}\right\rangle_{\partial K_{i}}=0, & \forall \mathbf{v} \in \mathbf{V}_{h}, \\ \left(\varepsilon_{r} \frac{\partial \mathbf{E}_{h}}{\partial t}, \mathbf{v}\right)_{K_{i}}-\left(\mathbf{H}_{h}, \text { curlv }\right)_{K_{i}}+\left\langle\mathbf{H}_{h}^{*} \times \mathbf{n}, \mathbf{v}\right\rangle_{\partial K_{i}}=0, \quad \forall \mathbf{v} \in \mathbf{V}_{h} .\end{cases}
$$

Here, $\mathbf{E}_{h}^{*}$ and $\mathbf{H}_{h}^{*}$ are the so-called numerical traces used to communicate information between adjacent elements, and $\mathbf{V}_{h}$ denotes the discontinuous finite element space

$$
\mathbf{V}_{h}=\left\{\mathbf{v} \in\left(L^{2}(\Omega)\right)^{3} \mid \mathbf{v}_{\mid K_{i}} \in\left(\mathbb{P}_{p_{i}}\left(K_{i}\right)\right)^{3}, \quad 1 \leq i \leq \mathcal{N}_{\Omega}\right\}
$$

where $\mathbb{P}_{p_{i}}\left(K_{i}\right)$ is the space of nodal polynomials of degree at most $p_{i}$ inside the element $K_{i}$. By gathering the electric and magnetic vectors DoF in each element into column vectors, we derive the following global semi-discrete system

$$
\left\{\begin{array}{l}
\mu_{r} \mathbf{M} \frac{\partial \underline{\mathbf{H}}_{h}}{\partial t}=-\mathbf{K} \underline{\mathbf{E}}_{h}+\mathbf{S}^{i} \underline{\mathbf{E}}_{h}+\mathbf{S}^{e} \underline{\mathbf{H}}_{h}+\mathbf{B}^{e}(t), \\
\varepsilon_{r} \mathbf{M} \frac{\partial \underline{\mathbf{E}}_{h}}{\partial t}=\mathbf{K} \underline{\mathbf{H}}_{h}-\mathbf{S}^{i} \underline{\mathbf{H}}_{h}-\mathbf{S}^{h} \underline{\mathbf{E}}_{h}-\mathbf{B}^{h}(t),
\end{array}\right.
$$

where $\mathbf{M}$ is the symmetric positive definite matrix, $\mathbf{K}$ and $\mathbf{S}^{i}$ are the symmetric matrices, and $\mathbf{S}^{e}$ and $\mathbf{S}^{h}$ are the skew-symmetric matrices. For detailed descriptions of these matrices see [16].

\section{Non-intrusive reduced-order modeling}

\subsection{Two-step proper orthogonal decomposition}

Given the parameter value sampling $\theta^{i} \in \mathcal{P}_{\hbar}^{t r}=\left\{\theta^{1}, \theta^{2}, \cdots, \theta^{\mathcal{N}_{p}}\right\} \subset \mathcal{P}$ with $\mathcal{P}_{\hbar}^{t r}$ being the traning parameter dataset and $\mathcal{N}_{p}$ being the corresponding number, the high fidelity solutions of (1) can be obtained by solving the DG scheme (5) with the $\mathrm{LF}_{2}$ time scheme. For the 
parameter $\theta^{i} \in \mathcal{P}_{\hbar}^{t r}$, the time trajectory matrix, for example, $\mathbf{A}_{\mathbf{E}}^{i}$, at the time steps $t^{\left(n_{i}\right)} \in$ $\mathcal{T}_{\hbar}^{t r}=\left\{t^{\left(n_{1}\right)}, t^{\left(n_{2}\right)}, \cdots, t^{\left(n_{\mathcal{N}_{t}}\right)}\right\} \subset \mathcal{T}$ is

$$
\mathbf{A}_{\mathbf{E}}^{i}=\left[\begin{array}{cccc}
\underline{\mathbf{E}}_{h, 1}^{\left(n_{1}\right)}\left(\theta^{i}\right) & \underline{\mathbf{E}}_{h, 1}^{\left(n_{2}\right)}\left(\theta^{i}\right) & \cdots & \underline{\mathbf{E}}_{h, 1}^{\left(n_{\mathcal{N}_{t}}\right)}\left(\theta^{i}\right) \\
\underline{\mathbf{E}}_{h, 2}^{\left(n_{1}\right)}\left(\theta^{i}\right) & \underline{\mathbf{E}}_{h, 2}^{\left(n_{2}\right)}\left(\theta^{i}\right) & \cdots & \underline{\mathbf{E}}_{h, 2}^{\left(n_{\mathcal{N}_{t}}\right)}\left(\theta^{i}\right) \\
\vdots & \vdots & \ddots & \vdots \\
\underline{\mathbf{E}}_{h, \mathcal{N}_{d}}^{\left(n_{1}\right)}\left(\theta^{i}\right) & \underline{\mathbf{E}}_{h, \mathcal{N}_{d}}^{\left(n_{2}\right)}\left(\theta^{i}\right) & \cdots & \underline{\mathbf{E}}_{h, \mathcal{N}_{d}}^{\left(n_{\mathcal{N}_{t}}\right)}\left(\theta^{i}\right)
\end{array}\right] \in \mathcal{R}^{\mathcal{N}_{d} \times \mathcal{N}_{t}}, i=1,2, \cdots, \mathcal{N}_{p},
$$

and then the snapshots matrix for all parameters in $\mathcal{P}_{\hbar}^{t r}$ is

$$
\mathbf{A}_{\mathbf{E}}=\left[\begin{array}{llll}
\mathbf{A}_{\mathbf{E}}^{1} & \mathbf{A}_{\mathbf{E}}^{2} & \cdots & \mathbf{A}_{\mathbf{E}}^{\mathcal{N}_{p}}
\end{array}\right] \in \mathcal{R}^{\mathcal{N}_{d} \times \mathcal{N}_{t p}},
$$

where $\mathcal{N}_{d}$ is the number of DoF of the DG method, and $\mathcal{N}_{t p}=\mathcal{N}_{t} \cdot \mathcal{N}_{p}$ is the total number of snapshots. Similarly, one can get the snapshots matrix $\mathbf{A}_{\mathbf{H}}$ for the magnetic field. Our goal is to select the optimal POD basis functions, which have the lowest dimension possible, while still maintaining a good approximation of the original dataset. The SVD method is an important tool to obtain an optimal representation for $\mathbf{A}_{\mathbf{u}}(\mathbf{u} \in\{\mathbf{E}, \mathbf{H}\})$. Let

$$
\mathbf{U}_{\mathbf{u}}^{T} \mathbf{A}_{\mathbf{u}} \mathbf{V}_{\mathbf{u}}=\left[\begin{array}{cc}
\Sigma_{r_{\mathbf{u}} \times r_{\mathbf{u}}}^{\mathbf{u}} & \mathbf{O}_{r_{\mathbf{u}} \times\left(\mathcal{N}_{t p}-r_{\mathbf{u}}\right)} \\
\mathbf{O}_{\left(\mathcal{N}_{d}-r_{\mathbf{u}}\right) \times r_{\mathbf{u}}} & \mathbf{O}_{\left(\mathcal{N}_{d}-r_{\mathbf{u}}\right) \times\left(\mathcal{N}_{t p}-r_{\mathbf{u}}\right)}
\end{array}\right], \quad \mathbf{u} \in\{\mathbf{E}, \mathbf{H}\}
$$

where $\mathbf{U}_{\mathbf{u}}$ and $\mathbf{V}_{\mathbf{u}}$ are $\mathcal{N}_{d} \times \mathcal{N}_{d}$ and $\mathcal{N}_{t p} \times \mathcal{N}_{t p}$ orthogonal matrices, $r_{\mathbf{u}}$ is the rank of $\mathbf{A}_{\mathbf{u}}$, and $\Sigma_{r_{\mathbf{u}} \times r_{\mathbf{u}}}^{\mathbf{u}}=\operatorname{diag}\left(\sigma_{\mathbf{u}, 1}, \sigma_{\mathbf{u}, 2}, \cdots, \sigma_{\mathbf{u}, r_{\mathbf{u}}}\right)$ with $\sigma_{\mathbf{u}, 1} \geq \sigma_{\mathbf{u}, 2} \geq \cdots \geq \sigma_{\mathbf{u}, r_{\mathbf{u}}}>0$ being the singular values of $\mathbf{A}_{\mathbf{u}}$. Let $\mathbf{U}_{\mathbf{u}}=\left(\phi_{\mathbf{u}, 1}, \phi_{\mathbf{u}, 2}, \cdots, \phi_{\mathbf{u}, \mathcal{N}_{d}}\right)$ and $\mathbf{V}_{\mathbf{u}}=\left(\varphi_{\mathbf{u}, 1}, \varphi_{\mathbf{u}, 2}, \cdots, \varphi_{\mathbf{u}, \mathcal{N}_{t p}}\right)$, the POD basis functions of dimension $k_{\mathbf{u}}\left(k_{\mathbf{u}} \ll r_{\mathbf{u}}\right)$ is the set $\left\{\phi_{\mathbf{u}, i}\right\}_{i=1}^{k_{\mathbf{u}}}$ in ROM, which can be used to construct the RB subspace $\mathcal{V}_{r b, \mathbf{u}}=\operatorname{span}\left\{\phi_{\mathbf{u}, 1}, \phi_{\mathbf{u}, 2}, \cdots, \phi_{\mathbf{u}, k_{\mathbf{u}}}\right\}$. In particular, according to Schmidt-Eckart-Young (S-E-Y) theorem $[44,45]$, the POD basis functions of rank $k_{\mathbf{u}}$ minimizes the projection error, i.e.,

$$
\begin{aligned}
\sum_{j=1}^{\mathcal{N}_{p}} \sum_{i=1}^{\mathcal{N}_{t}}\left\|\mathbf{A}_{\mathbf{u}}^{j}(:, i)-\Xi_{\mathbf{u}} \Xi_{\mathbf{u}}^{T} \mathbf{A}_{\mathbf{u}}^{j}(:, i)\right\|_{\mathcal{R}^{\mathcal{N}_{d}}}^{2} & =\min _{\Upsilon_{\mathbf{u}} \in \prod_{k \mathbf{u}}} \sum_{j=1}^{\mathcal{N}_{p}} \sum_{i=1}^{\mathcal{N}_{t}}\left\|\mathbf{A}_{\mathbf{u}}^{j}(:, i)-\Upsilon_{\mathbf{u}} \Upsilon_{\mathbf{u}}^{T} \mathbf{A}_{\mathbf{u}}^{j}(:, i)\right\|_{\mathcal{R}^{\mathcal{N}_{d}}}^{2} \\
& =\sum_{i=k_{\mathbf{u}}+1}^{r_{\mathbf{u}}} \sigma_{\mathbf{u}, i}^{2}, \quad \mathbf{u} \in\{\mathbf{E}, \mathbf{H}\},
\end{aligned}
$$

where $\Xi_{\mathbf{u}}=\left\{\phi_{\mathbf{u}, 1}, \phi_{\mathbf{u}, 2}, \cdots, \phi_{\mathbf{u}, k_{\mathbf{u}}}\right\}$ is the basis matrix comprising the first $k_{\mathbf{u}}$ left singular vectors of $\mathbf{A}_{\mathbf{u}}, \mathbf{A}_{\mathbf{u}}^{j}(:, i)$ is the $i$-th column of $\mathbf{A}_{\mathbf{u}}^{j}\left(j=1,2, \cdots, \mathcal{N}_{p}\right)$, and $\prod_{k_{\mathbf{u}}}=\left\{\Upsilon_{\mathbf{u}} \in \mathcal{R}^{\mathcal{N}_{d} \times k_{\mathbf{u}}}\right.$ : $\left.\Upsilon_{\mathbf{u}}^{T} \Upsilon_{\mathbf{u}}=\mathbf{I}_{k_{\mathbf{u}}}\right\}$ is the set of all $k_{\mathbf{u}}$-dimensional orthogonal basis functions. In addition, one can choose $k_{\mathbf{u}}$ to be the smallest integer such that

$$
k_{\mathbf{u}}=\operatorname{argmin}\left\{\mathcal{E}\left(k_{\mathbf{u}}\right): \mathcal{E}\left(k_{\mathbf{u}}\right) \geq 1-\rho\right\}, \quad \mathbf{u} \in\{\mathbf{E}, \mathbf{H}\},
$$

with $\mathcal{E}\left(k_{\mathbf{u}}\right)=\sum_{i=1}^{k_{\mathbf{u}}} \sigma_{\mathbf{u}, i}^{2} / \sum_{i=1}^{r_{\mathbf{u}}} \sigma_{\mathbf{u}, i}^{2}$ being the relative information content, where $\rho$ is the truncation tolerance.

It can be shown that computing the POD basis function is equivalent to solve the following eigenvalue problem [46]

$$
\mathbf{C}_{\mathbf{u}} \varphi_{\mathbf{u}, i}=\lambda_{\mathbf{u}, i} \varphi_{\mathbf{u}, i}, \quad i=1,2, \cdots, r_{\mathbf{u}}, \mathbf{u} \in\{\mathbf{E}, \mathbf{H}\},
$$


where $\mathbf{C}_{\mathbf{u}}=\mathbf{A}_{\mathbf{u}}^{T} \mathbf{A}_{\mathbf{u}} \in \mathcal{R}^{\mathcal{N}_{t p} \times \mathcal{N}_{t p} p}$ is the correlation matrix of snapshot matrix $\mathbf{A}_{\mathbf{u}}$, and $\lambda_{\mathbf{u}, i}=\sigma_{\mathbf{u}, i}^{2}$ is the eigenvalues of $\mathbf{C}_{\mathbf{u}}$. One can then compute the POD basis function [26] as follows

$$
\phi_{\mathbf{u}, i}=\frac{1}{\sqrt{\lambda_{\mathbf{u}, i}}} \mathbf{A}_{\mathbf{u}} \varphi_{\mathbf{u}, i}, \quad i=1,2, \cdots, k_{\mathbf{u}}, k_{\mathbf{u}} \ll r_{\mathbf{u}}, \mathbf{u} \in\{\mathbf{E}, \mathbf{H}\} .
$$

Moreover, the standard POD method described by (10)-(12) is not directly applied to obtain the orthogonal basis functions for the parameterized time-domain electromagnetic scattering problems due to the large size $\mathcal{N}_{t p}$ of correlation matrix $\mathbf{C}_{\mathbf{u}}$, making eigenvalue decomposition of $\mathbf{C}_{\mathbf{u}}$ very expensive. Instead, we use a two-step or nested POD method [18, 47] described as follows, where the POD method is implemented separately for time and parameter spaces:

- For the parameter $\theta^{j} \in \mathcal{P}_{\hbar}^{t r}$, the $k_{\mathbf{u}}^{j}$ orthogonal basis functions $\phi_{\mathbf{u}, i}^{j}\left(i=1,2, \cdots, k_{\mathbf{u}}^{j}\right)$ are obtained by using the POD method (10)-(12) with the truncation tolerance $\rho_{t}$ for the time trajectory matrix $\mathbf{A}_{\mathbf{u}}^{j}(\mathbf{u} \in\{\mathbf{E}, \mathbf{H}\})$, i.e., $\Phi_{\mathbf{u}}^{j}=\left(\phi_{\mathbf{u}, 1}^{j}, \phi_{\mathbf{u}, 2}^{j}, \cdots, \phi_{\mathbf{u}, k_{\mathbf{u}}^{j}}^{j}\right)$.

- With the truncation tolerance $\rho_{\theta}$, the $L_{\mathbf{u}}$ orthogonal basis functions $\phi_{\mathbf{u}, i}\left(i=1,2, \cdots, L_{\mathbf{u}}\right)$ are obtained by using the POD method (10)-(12) for the matrix $\Lambda_{\mathbf{u}}=\left(\Phi_{\mathbf{u}}^{1}, \Phi_{\mathbf{u}}^{2}, \cdots, \Phi_{\mathbf{u}}^{\mathcal{N}_{p}}\right)$, i.e., $\Phi_{\mathbf{u}}=\left(\phi_{\mathbf{u}, 1}, \phi_{\mathbf{u}, 2}, \cdots, \phi_{\mathbf{u}, L_{\mathbf{u}}}\right)$.

Algorithm 1 summarizes the two-step POD method used to obtain the basis functions.

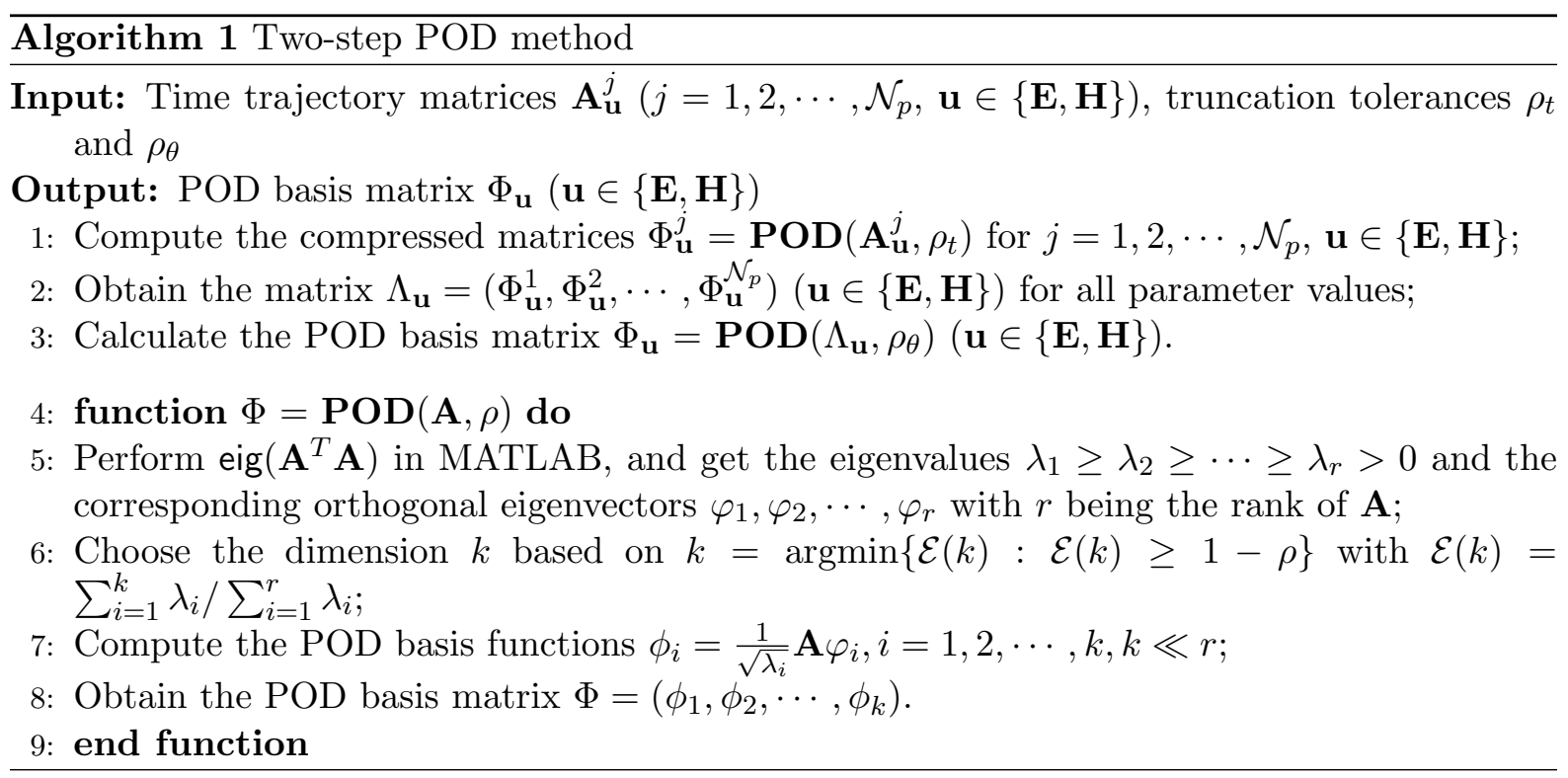

Remark 1. The snapshot vectors in this paper are chosen from the high fidelity DGTD simulations. However, one may obtain the snapshots from physical system trajectories by drawing samples from experiments and interpolation (or data assimilation). In particular, for the sake of simplicity, we equidistantly extract $\mathcal{N}_{t}$ transient solutions after the electric and magnetic values are stable for the sampling parameter $\theta_{i} \in \mathcal{P}_{\hbar}^{\text {tr }}$ in this study, because we pay more attention to the solutions in the frequency-domain and the calculation of the radar cross section (RCS) for the electromagnetic scattering problems, which is only related to the stable time-domain electromagnetic fields.

Remark 2. The computational costs of the matrix-matrix multiplication ( $M-M)$, the eigenvalue calculation (E-C) and the matrix-vector $(M-C)$ multiplication in the two-step POD method are 
listed in Table 1, in which $\mathcal{N}_{k_{\mathbf{u}}}=\sum_{j=1}^{\mathcal{N}_{p}} k_{\mathbf{u}}^{j}$, and we assume $\mathcal{N}_{k_{\mathbf{u}}} \approx \mathcal{N}_{t}$. Besides, the corresponding complexities in one-step POD method are also shown. From Table 1, we deduce that the two-step POD method is a good candidate to save CPU time.

Table 1: Comparison of the complexities between the standard POD and two-step POD methods.

\begin{tabular}{cccc}
\hline Method & M-M & E-C & M-C \\
\hline POD & $\mathcal{O}\left(\mathcal{N}_{t}^{2} \mathcal{N}_{p}^{2} \mathcal{N}_{d}\right)$ & $\mathcal{O}\left(\mathcal{N}_{t}^{3} \mathcal{N}_{p}^{3}\right)$ & $\mathcal{O}\left(\mathcal{N}_{d} \mathcal{N}_{t} \mathcal{N}_{p} L_{\mathbf{u}}\right)$ \\
\hline First step & $\mathcal{O}\left(\mathcal{N}_{t}^{2} \mathcal{N}_{d}\right) \times \mathcal{N}_{p}$ & $\mathcal{O}\left(\mathcal{N}_{t}^{3}\right) \times \mathcal{N}_{p}$ & $\sum_{j=1}^{\mathcal{N}_{p}}\left(\mathcal{O}\left(\mathcal{N}_{d} \mathcal{N}_{t} k_{\mathbf{u}}^{j}\right)\right)$ \\
Second step & $\mathcal{O}\left(\mathcal{N}_{k_{\mathbf{u}}}^{2} \mathcal{N}_{d}\right)$ & $\mathcal{O}\left(\mathcal{N}_{k_{\mathbf{u}}}^{3}\right)$ & $\mathcal{O}\left(\mathcal{N}_{d} \mathcal{N}_{k_{\mathbf{u}}} L_{\mathbf{u}}\right)$ \\
Two-step POD $(\approx)$ & $\mathcal{O}\left(\mathcal{N}_{t}^{2} \mathcal{N}_{d}\right) \times\left(\mathcal{N}_{p}+1\right)$ & $\mathcal{O}\left(\mathcal{N}_{t}^{3}\right) \times\left(\mathcal{N}_{p}+1\right)$ & $\mathcal{O}\left(\mathcal{N}_{d} \mathcal{N}_{t}\left(L_{\mathbf{u}}+\mathcal{N}_{t}\right)\right)$ \\
\hline
\end{tabular}

Remark 3. Based on (9) and (10), the projection errors in the first and second steps of the two-step POD method respectively are

$$
\sum_{i=1}^{\mathcal{N}_{t}}\left\|\mathbf{A}_{\mathbf{u}}^{j}(:, i)-\Phi_{\mathbf{u}}^{j}\left(\Phi_{\mathbf{u}}^{j}\right)^{T} \mathbf{A}_{\mathbf{u}}^{j}(:, i)\right\|_{\mathcal{R}^{\mathcal{N}_{d}}}^{2} \leq \rho_{t} \sum_{i=1}^{r_{\mathbf{u}}^{j}}\left(\sigma_{\mathbf{u}, i}^{j}\right)^{2}, \quad j=1,2, \cdots, \mathcal{N}_{p},
$$

and

$$
\sum_{j=1}^{\mathcal{N}_{p}} \sum_{i=1}^{k_{\mathbf{u}}^{j}}\left\|\Phi_{\mathbf{u}}^{j}(:, i)-\Phi_{\mathbf{u}} \Phi_{\mathbf{u}}^{T} \Phi_{\mathbf{u}}^{j}(:, i)\right\|_{\mathcal{R}^{\mathcal{N}_{d}}}^{2} \leq \rho_{\theta} \sum_{i=1}^{R_{\mathbf{u}}} \iota_{\mathbf{u}, i}^{2} .
$$

Here, $\mathbf{A}_{\mathbf{u}}^{j}(:, i)$ and $\Phi_{\mathbf{u}}^{j}(:, i)$ respectively are the $i$-th column of matrices $\mathbf{A}_{\mathbf{u}}^{j}$ and $\Phi_{\mathbf{u}}^{j},\left\{\sigma_{\mathbf{u}, i}^{j}\right\}_{i=1}^{r_{\mathbf{u}}^{j}}$ $\left(j=1,2, \cdots, \mathcal{N}_{p}\right),\left\{\iota_{\mathbf{u}, i}\right\}_{i=1}^{R_{\mathbf{u}}}$ respectively are the singular values of $\mathbf{A}_{\mathbf{u}}^{j}$ and $\Lambda_{\mathbf{u}}, r_{\mathbf{u}}^{j}$ and $R_{\mathbf{u}}$ are the corresponding ranks, and $\rho_{t}$ and $\rho_{\theta}$ are the truncation tolerances. The projection error bound of the snapshot vectors $\mathbf{A}_{\mathbf{u}}$ onto the $R B$ subspace $\mathcal{V}_{r b, \mathbf{u}}$ then is

$$
\sum_{j=1}^{\mathcal{N}_{p}} \sum_{i=1}^{\mathcal{N}_{t}}\left\|\mathbf{A}_{\mathbf{u}}^{j}(:, i)-\Phi_{\mathbf{u}} \Phi_{\mathbf{u}}^{T} \mathbf{A}_{\mathbf{u}}^{j}(:, i)\right\|_{\mathcal{R}^{\mathcal{N}_{d}}} \leq \rho_{t}^{\frac{1}{2}} \mathcal{M}_{1}+\rho_{\theta}^{\frac{1}{2}} \mathcal{M}_{2}, \quad \mathbf{u} \in\{\mathbf{E}, \mathbf{H}\}
$$

with

$$
\left\{\begin{array}{l}
\mathcal{M}_{1}=\mathcal{N}_{t}^{\frac{1}{2}}\left(1+\chi_{\max }\left(\Phi_{\mathbf{u}} \Phi_{\mathbf{u}}^{T}\right)\right) \sum_{j=1}^{\mathcal{N}_{p}}\left(\sum_{i=1}^{r_{\mathbf{u}}^{j}}\left(\sigma_{\mathbf{u}, i}^{j}\right)^{2}\right)^{\frac{1}{2}}, \\
\mathcal{M}_{2}=\mathcal{N}_{k_{\mathbf{u}}}^{\frac{1}{2}} \max _{1 \leq j \leq \mathcal{N}_{p}} \sum_{i=1}^{\mathcal{N}_{t}}\left\|\mathbf{A}_{\mathbf{u}}^{j}(:, i)\right\|_{\mathcal{R}^{\mathcal{N}_{d}}}\left(\sum_{i=1}^{R_{\mathbf{u}}}\left(\iota_{\mathbf{u}, i}\right)^{2}\right)^{\frac{1}{2}},
\end{array}\right.
$$

where $\chi_{\max }\left(\Phi_{\mathbf{u}} \Phi_{\mathbf{u}}^{T}\right)$ denotes the maximum singular value of matrix $\Phi_{\mathbf{u}} \Phi_{\mathbf{u}}^{T}$, and $\mathcal{N}_{k_{\mathbf{u}}}=\sum_{j=1}^{\mathcal{N}_{p}} k_{\mathbf{u}}^{j}$.

\subsection{Approximation of the reduced-order coefficients}

For the electromagnetic scattering problems, the reduced-order solution $\underline{\mathbf{u}}_{h}^{r}(\mathbf{u} \in\{\mathbf{E}, \mathbf{H}\})$ for a certain time and parameter values $(t, \theta) \in \mathcal{T} \times \mathcal{P}$ can be understood as the projection of the high fidelity $\underline{\mathbf{u}}_{h}$ solution on the RB subspace $\mathcal{V}_{r b, \mathbf{u}}$ in the MOR method. According to the projection theory, one can obtain the reduced-order solution and the corresponding reducedorder or projection coefficient

$$
\left\{\begin{array}{l}
\underline{\mathbf{u}}_{h}^{r}(t, \theta)=\Phi_{\mathbf{u}}\left(\Phi_{\mathbf{u}}^{T} \Phi_{\mathbf{u}}\right)^{-1} \Phi_{\mathbf{u}}^{T} \underline{\mathbf{u}}_{h}(t, \theta)=\Phi_{\mathbf{u}} \Phi_{\mathbf{u}}^{T} \underline{\mathbf{u}}_{h}(t, \theta), \\
\alpha_{\mathbf{u}}(t, \theta)=\left(\Phi_{\mathbf{u}}^{T} \Phi_{\mathbf{u}}\right)^{-1} \Phi_{\mathbf{u}}^{T} \underline{\mathbf{u}}_{h}(t, \theta)=\Phi_{\mathbf{u}}^{T} \underline{\mathbf{u}}_{h}(t, \theta),
\end{array} \quad \mathbf{u} \in\{\mathbf{E}, \mathbf{H}\} .\right.
$$


A standard Galerkin approach is used to determine the reduced-order coefficient in the conventional framework of the MOR method [2]. However, the major drawback of the MOR method is that they require access to the HDM [30], which may be provide limited computational gain especially for complex nonlinear problems and suffer from instabilities [18]. So, we introduce the non-intrusive MOR method in this study, where the mappings between the time/parameter valuers and the reduced-order coefficients are approximated.

During the offline stage, in order to obtain the projection coefficient $\alpha_{\mathbf{u}}\left(t^{*}, \theta^{*}\right)$ for new time/parameter values $\left(t^{*}, \theta^{*}\right) \in \mathcal{T} \times \mathcal{P}$, an ideal mapping $\varpi_{\mathbf{u}}$ from an input vector $(t, \theta)$ of dimension $\operatorname{dim}(\mathcal{P})+1=d+1$ to an output vector $\alpha_{\mathbf{u}}(t, \theta)$ of dimension $L_{\mathbf{u}}$ should be built

$$
\varpi_{\mathbf{u}}:(t, \theta) \rightarrow \alpha_{\mathbf{u}}(t, \theta), \quad \mathbf{u} \in\{\mathbf{E}, \mathbf{H}\} .
$$

In this paper, we use the cubic spline interpolation (CSI) technique to construct an approximation $\widehat{\varpi}_{\mathbf{u}}$ of the mapping $\varpi_{\mathbf{u}}$ via a set of training data

$$
\begin{aligned}
\mathcal{T}_{\hbar}^{t r} & =\left\{t^{\left(n_{1}\right)}, t^{\left(n_{2}\right)}, \cdots, t^{\left(n_{\mathcal{N}_{t}}\right)}\right\} \subset \mathcal{T} \\
\mathcal{P}_{\hbar}^{t r} & =\left\{\theta^{1}, \theta^{2}, \cdots, \theta^{\mathcal{N}_{p}}\right\} \subset \mathcal{P} \\
\mathcal{D}_{\mathbf{u}, \hbar}^{t r} & =\left\{\alpha_{\mathbf{u}}(t, \theta)=\Phi_{\mathbf{u}}^{T} \underline{\mathbf{u}}_{h}(t, \theta): t \in \mathcal{T}_{\hbar}^{t r}, \theta \in \mathcal{P}_{\hbar}^{t r}\right\} \subset \mathcal{R}^{L_{\mathbf{u}}}, \mathbf{u} \in\{\mathbf{E}, \mathbf{H}\} .
\end{aligned}
$$

During the online stage, the approximate projection coefficient $\widehat{\alpha}_{\mathbf{u}}\left(t^{*}, \theta^{*}\right)$ for the new time and parameter values $\left(t^{*}, \theta^{*}\right) \in \mathcal{T} \times \mathcal{P}$ can be obtained as

$$
\widehat{\alpha}_{\mathbf{u}}\left(t^{*}, \theta^{*}\right)=\widehat{\varpi}_{\mathbf{u}}\left(t^{*}, \theta^{*}\right), \mathbf{u} \in\{\mathbf{E}, \mathbf{H}\},
$$

and then the reduced-order solution $\underline{\mathbf{u}}_{h}^{r}\left(t^{*}, \theta^{*}\right)$ can be calculated as

$$
\underline{\mathbf{u}}_{h}^{r}\left(t^{*}, \theta^{*}\right)=\Phi_{\mathbf{u}} \widehat{\alpha}_{\mathbf{u}}\left(t^{*}, \theta^{*}\right) \in \mathcal{V}_{r b, \mathbf{u}}, \mathbf{u} \in\{\mathbf{E}, \mathbf{H}\} .
$$

Algorithm 2 describes the implementation of the offline and online stages of the non-intrusive MOR method.

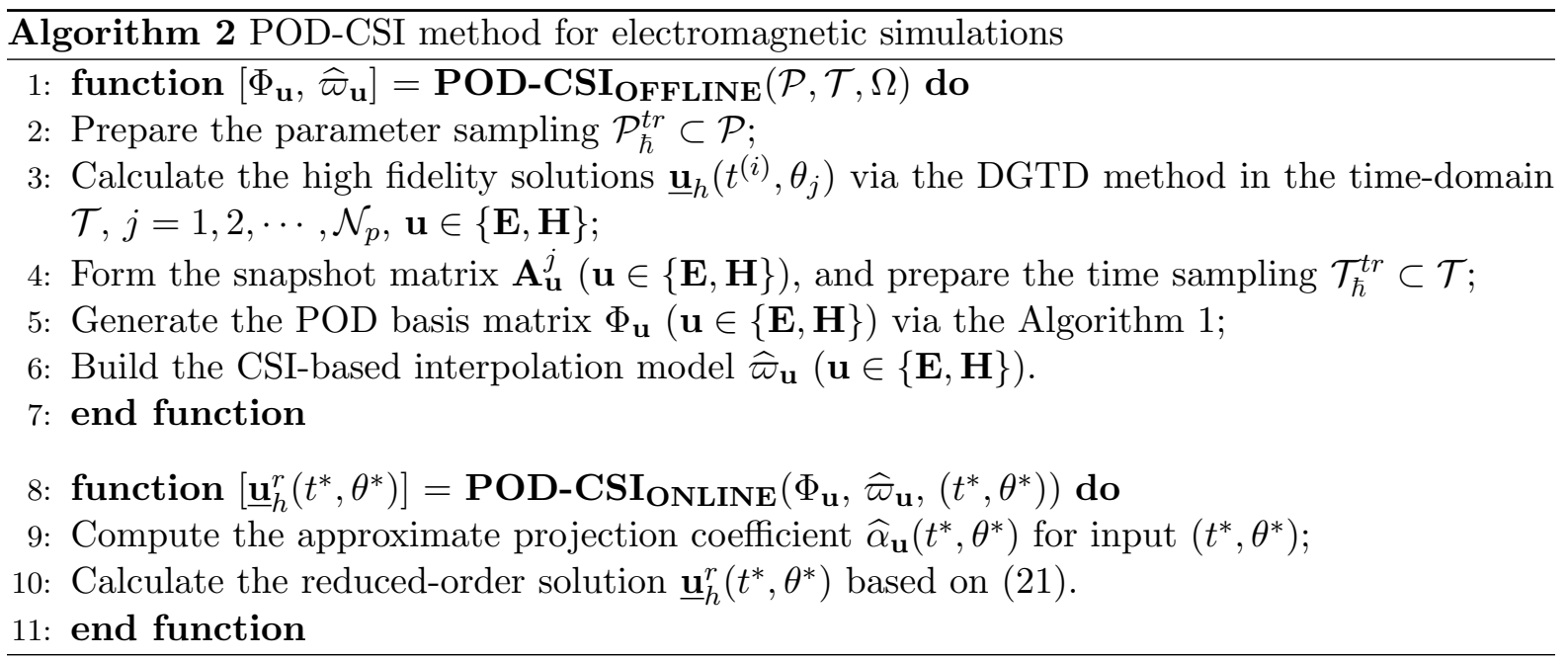

Remark 4. In Algorithm 2, we find that the offline and online stages of the POD-CSI method are completely decoupled. The CSI-based interpolation models and POD basis functions can be saved during offline stage, and they can be used directly for a desired time and parameter values $\left(t^{*}, \theta^{*}\right) \in \mathcal{T} \times \mathcal{P}$ during online stage. 


\section{Cubic spline interpolation models}

In this section, we will briefly introduce the definition of CSI method and the structure of the CSI-based approximation of reduced-order coefficient matrices.

\subsection{Cubic spline interpolation}

Given the interpolation nodes $a=x_{0}<x_{1}<\cdots<x_{n}=b$, and the corresponding function values $f\left(x_{k}\right)=y_{k}, k=0,1,2 \cdots, n$, if $S(x)$ satisfies the following three conditions [48]

$$
\left\{\begin{array}{l}
S(x) \in \mathcal{C}^{2}[a, b] \\
S\left(x_{k}\right)=f\left(x_{k}\right)=y_{k}, k=0,1,2 \cdots, n \\
S(x) \text { is the cubic polynomial in }\left[x_{k}, x_{k+1}\right], k=0,1,2, \cdots, n-1,
\end{array}\right.
$$

then $S(x)$ is said to be the cubic spline function of $f(x)$. Let $S_{k}(x)$ be the expression of $S(x)$ in $\left[x_{k}, x_{k+1}\right], k=0,1,2 \cdots, n-1$, which contains $4 n$ unknowns, and then based on (22), we have the interpolation conditions

$$
S_{k}\left(x_{k}\right)=y_{k}, S_{k}\left(x_{k+1}\right)=y_{k+1}, k=0,1,2, \cdots, n-1,
$$

and the differential continuity conditions

$$
S_{k-1}^{\prime}\left(x_{k}^{-}\right)=S_{k}^{\prime}\left(x_{k}^{+}\right), S_{k-1}^{\prime \prime}\left(x_{k}^{-}\right)=S_{k}^{\prime \prime}\left(x_{k}^{+}\right), k=1,2, \cdots, n-1,
$$

which includes $2 n+2(n-1)=4 n-2$ conditions. Based on (23) and (24), one cannot obtain the cubic spline function $S(x)$, so we have to add two other boundary conditions. In this study, we consider the not-a-knot conditions [49]

$$
S_{0}^{\prime \prime \prime}\left(x_{1}^{-}\right)=S_{1}^{\prime \prime \prime}\left(x_{1}^{+}\right), S_{n-2}^{\prime \prime \prime}\left(x_{n-1}^{-}\right)=S_{n-1}^{\prime \prime \prime}\left(x_{n-1}^{+}\right) .
$$

Combining the conditions (23)-(25), one can get the cubic polynomial $S_{k}(x)(k=0,1, \cdots, n-1)$ by solving a $(n+1)$ order system of linear equations. In particular, one can use the widely used tensor product formulation to extend the same analysis of multiple independent variables $[50,51]$. To determine the interpolated value at a desired point, we use cubic interpolation of the values at closest knot points in each respective dimension. The number of independent variables is not limited in multivariate CSI method. For more detailed definition of multivariate CSI method please see [52].

\subsection{CSI-based approximation of reduced-order coefficient matrices}

To better capture the relationship between the time/parameter values $(t, \theta)$ and the projection coefficients $\alpha_{\mathbf{u}}^{j}(t, \theta)\left(\forall(t, \theta) \in \mathcal{T}_{\hbar}^{t r} \times \mathcal{P}_{\hbar}^{t r}\right)$ with the $\alpha_{\mathbf{u}}^{j}(t, \theta)$ being the $j$-th component of $\alpha_{\mathbf{u}}(t, \theta)$, we rewrite the training dataset $\mathcal{D}_{\mathbf{u}, \hbar}^{t r}$ in matrix forms

$$
\begin{aligned}
\mathbf{Q}_{\mathbf{u}}^{j} & =\left[\begin{array}{cccc}
\alpha_{\mathbf{u}}^{j}\left(t^{\left(n_{1}\right)}, \theta^{1}\right) & \alpha_{\mathbf{u}}^{j}\left(t^{\left(n_{1}\right)}, \theta^{2}\right) & \cdots & \alpha_{\mathbf{u}}^{j}\left(t^{\left(n_{1}\right)}, \theta^{\mathcal{N}_{p}}\right) \\
\alpha_{\mathbf{u}}^{j}\left(t^{\left(n_{2}\right)}, \theta^{1}\right) & \alpha_{\mathbf{u}}^{j}\left(t^{\left(n_{2}\right)}, \theta^{2}\right) & \cdots & \alpha_{\mathbf{u}}^{j}\left(t^{\left(n_{2}\right)}, \theta^{\mathcal{N}_{p}}\right) \\
\vdots & \vdots & \ddots & \vdots \\
\alpha_{\mathbf{u}}^{j}\left(t^{\left(n_{\mathcal{N}_{t}}\right)}, \theta^{1}\right) & \alpha_{\mathbf{u}}^{j}\left(t^{\left(n_{\mathcal{N}_{t}}\right)}, \theta^{2}\right) & \cdots & \alpha_{\mathbf{u}}^{j}\left(t^{\left(n_{\mathcal{N}_{t}}\right)}, \theta^{\mathcal{N}_{p}}\right)
\end{array}\right] \\
& =\left[\phi_{\mathbf{u}, j}^{T} \mathbf{A}_{\mathbf{u}}^{1} ; \phi_{\mathbf{u}, j}^{T} \mathbf{A}_{\mathbf{u}}^{2} ; \cdots ; \phi_{\mathbf{u}, j}^{T} \mathbf{A}_{\mathbf{u}}^{\mathcal{N}_{p}}\right]^{T} \in \mathcal{R}^{\mathcal{N}_{t} \times \mathcal{N}_{p}}, j=1,2, \cdots, L_{\mathbf{u}}, \mathbf{u} \in\{\mathbf{E}, \mathbf{H}\} .
\end{aligned}
$$

Let

$$
\mathbf{Q}_{\mathbf{u}}^{j} \approx \widetilde{\mathbf{Q}}_{\mathbf{u}}^{j}=\sum_{i=1}^{l_{\mathbf{u}}^{j}} \delta_{\mathbf{u}, i}^{j} \zeta_{\mathbf{u}, i}^{j}\left(\eta_{\mathbf{u}, i}^{j}\right)^{T}, j=1,2, \cdots, L_{\mathbf{u}}, \mathbf{u} \in\{\mathbf{E}, \mathbf{H}\}
$$


where $\zeta_{\mathbf{u}, i}^{j}$ and $\eta_{\mathbf{u}, i}^{j}$ respectively are the $i$-th discrete time- and parameter-modes of $j$-th projection coefficient, $\delta_{\mathbf{u}, 1}^{j} \geq \delta_{\mathbf{u}, 2}^{j} \geq \cdots \geq \delta_{\mathbf{u}, l_{\mathbf{u}}^{j}}^{j}>0$ are the first $l_{\mathbf{u}}^{j}$ singular values of matrix $\mathbf{Q}_{\mathbf{u}}^{j}$, and $l_{\mathbf{u}}^{j}$ is the truncation rank obtained by $l_{\mathbf{u}}^{j}=\operatorname{argmin}\left\{\mathcal{S}\left(l_{\mathbf{u}}^{j}\right): \mathcal{S}\left(l_{\mathbf{u}}^{j}\right) \geq 1-\rho_{l, \text { tol }}\right\}$ with $\mathcal{S}\left(l_{\mathbf{u}}^{j}\right)=\sum_{i=1}^{l_{\mathbf{u}}^{j}}\left(\delta_{\mathbf{u}, i}^{j}\right)^{2} / \sum_{i=1}^{r_{\mathbf{u}, q}^{j}}\left(\delta_{\mathbf{u}, i}^{j}\right)^{2}$ being the relative information content, where $r_{\mathbf{u}, q}^{j}$ is the rank of $\mathbf{Q}_{\mathbf{u}}^{j}$, and $\rho_{l, \text { tol }}$ is the truncation tolerance. Now, for the $i$-th discrete time and parameter modes of $j$-th projection coefficient, one can use the CSI method to construct the corresponding continuous time- and parameter-modes, i.e.,

$$
\left\{\begin{aligned}
\left\{\left(t^{\left(n_{k}\right)},\left(\zeta_{\mathbf{u}, i}^{j}\right)_{k}\right), k=1,2, \cdots, \mathcal{N}_{t}\right\} & \stackrel{\text { CSI-based approximation }}{\longrightarrow}\left\{\left(t, \widehat{\zeta}_{\mathbf{u}, i}^{j}(t)\right)\right\}, \\
\left\{\left(\theta^{k},\left(\eta_{\mathbf{u}, i}^{j}\right)_{k}\right), k=1,2, \cdots, \mathcal{N}_{p}\right\} & \stackrel{\text { CSI-based approximation }}{\longrightarrow}\left\{\left(\theta, \widehat{\eta}_{\mathbf{u}, i}^{j}(\theta)\right)\right\},
\end{aligned}\right.
$$

where the values $\left(\zeta_{\mathbf{u}, i}^{j}\right)_{k}$ and $\left(\eta_{\mathbf{u}, i}^{j}\right)_{k}$ respectively are the $k$-th component of vectors $\zeta_{\mathbf{u}, i}^{j}$ and $\eta_{\mathbf{u}, i}^{j}$. Based on (26)-(28), we can get the approximation of the reduced-order coefficient for a new time/parameter values $\left(t^{*}, \theta^{*}\right)$

$$
\alpha_{\mathbf{u}}^{j}\left(t^{*}, \theta^{*}\right) \approx \widetilde{\alpha}_{\mathbf{u}}^{j}\left(t^{*}, \theta^{*}\right) \approx \widehat{\alpha}_{\mathbf{u}}^{j}\left(t^{*}, \theta^{*}\right)=\sum_{i=1}^{l_{\mathbf{u}}^{j}} \delta_{\mathbf{u}, i}^{j} \widehat{\zeta}_{\mathbf{u}, i}^{j}\left(t^{*}\right) \widehat{\eta}_{\mathbf{u}, i}^{j}\left(\theta^{*}\right), \mathbf{u} \in\{\mathbf{E}, \mathbf{H}\},
$$

where $\widehat{\alpha}_{\mathbf{u}}^{j}\left(t^{*}, \theta^{*}\right)$ is the approximation of reduced-order coefficient via the CSI method, $\widetilde{\alpha}_{\mathbf{u}}^{j}\left(t^{*}, \theta^{*}\right)$ is the approximation of reduced-order coefficient via the SVD method. Combining (21), one can obtain the reduced-order solution by using the POD-CSI method.

Remark 5. Assuming that all samples have been used both as snapshots for constructing the $P O D$ basis and as training samples of the interpolation model, the total error between the projection solutions $\underline{\mathbf{u}}_{h}^{p}\left(t^{\left(n_{i}\right)}, \theta^{k}\right)=\Phi_{\mathbf{u}} \Phi_{\mathbf{u}}^{T} \underline{\mathbf{u}}_{h}\left(t^{\left(n_{i}\right)}, \theta^{k}\right)$ and the POD-CSI reduced-order solutions $\underline{\mathbf{u}}_{h}^{r}\left(t^{\left(n_{i}\right)}, \theta^{k}\right)\left(\left(t^{\left(n_{i}\right)}, \theta^{k}\right) \in \mathcal{T}_{\hbar}^{t r} \times \mathcal{P}_{\hbar}^{t r}\right)$ can be expressed as

$$
\begin{aligned}
\sum_{k=1}^{\mathcal{N}_{p}} \sum_{i=1}^{\mathcal{N}_{t}}\left\|\underline{\mathbf{u}}_{h}^{p}\left(t^{\left(n_{i}\right)}, \theta^{k}\right)-\underline{\mathbf{u}}_{h}^{r}\left(t^{\left(n_{i}\right)}, \theta^{k}\right)\right\|_{\mathcal{R}^{\mathcal{N}_{d}}}^{2} & =\sum_{k=1}^{\mathcal{N}_{p}} \sum_{i=1}^{\mathcal{N}_{t}}\left\|\sum_{j=1}^{L_{\mathbf{u}}} \phi_{\mathbf{u}, j}\left(\mathbf{Q}_{\mathbf{u}}^{j}\right)_{i k}-\sum_{j=1}^{L_{\mathbf{u}}} \phi_{\mathbf{u}, j}\left(\widehat{\mathbf{Q}}_{\mathbf{u}}^{j}\right)_{i k}\right\|_{\mathcal{R}^{\mathcal{N}_{d}}}^{2} \\
& =\sum_{k=1}^{\mathcal{N}_{p}} \sum_{i=1}^{\mathcal{N}_{t}}\left\|\sum_{j=1}^{L_{\mathbf{u}}} \phi_{\mathbf{u}, j}\left(\mathbf{Q}_{\mathbf{u}}^{j}\right)_{i k}-\sum_{j=1}^{L_{\mathbf{u}}} \phi_{\mathbf{u}, j}\left(\widetilde{\mathbf{Q}}_{\mathbf{u}}^{j}\right)_{i k}\right\|_{\mathcal{R}^{\mathcal{N}_{d}}}^{2} \\
& \left.=\sum_{k=1}^{\mathcal{N}_{p}} \sum_{i=1}^{\mathcal{N}_{t}} \sum_{j=1}^{L_{\mathbf{u}}}\left(\left(\mathbf{Q}_{\mathbf{u}}^{j}\right)_{i k}-\widetilde{\mathbf{Q}}_{\mathbf{u}}^{j}\right)_{i k}\right)^{2} \\
& =\sum_{j=1}^{L_{\mathbf{u}}}\left\|\mathbf{Q}_{\mathbf{u}}^{j}-\widetilde{\mathbf{Q}}_{\mathbf{u}}^{j}\right\|_{F}^{2} \\
& \leq \sum_{j=1}^{L_{\mathbf{u}}}\left\|\mathbf{Q}_{\mathbf{u}}^{j}\right\|_{F}^{2} \rho_{j, t o l}^{2},
\end{aligned}
$$

where $\|\cdot\|_{F}$ denotes the Frbenius norm of a matrix, $\left(\widehat{\mathbf{Q}}_{\mathbf{u}}^{j}\right)_{i k}=\widehat{\alpha}_{\mathbf{u}}^{j}\left(t^{\left(n_{i}\right)}, \theta^{k}\right)$, and $\frac{\left\|\mathbf{Q}_{\mathbf{u}}^{j}-\widetilde{\mathbf{Q}}_{\mathbf{u}}^{j}\right\|_{F}^{2}}{\left\|\mathbf{Q}_{\mathbf{u}}^{j}\right\|_{F}^{2}} \leq$ $\rho_{j, t o l}^{2}\left(j=1,2, \cdots, L_{\mathbf{u}}\right)$ is the truncation error of $S V D$ method for the $j$-th projection coefficient, 
and then the total recovery error of the POD-CSI method can be estimated as

$$
\begin{aligned}
\sum_{k=1}^{\mathcal{N}_{p}} \sum_{i=1}^{\mathcal{N}_{t}}\left\|\underline{\mathbf{u}}_{h}\left(t^{\left(n_{i}\right)}, \theta^{k}\right)-\underline{\mathbf{u}}_{h}^{r}\left(t^{\left(n_{i}\right)}, \theta^{k}\right)\right\|_{\mathcal{R}^{\mathcal{N}_{d}}} \leq & \sum_{k=1}^{\mathcal{N}_{p}} \sum_{i=1}^{\mathcal{N}_{t}}\left\|\underline{\mathbf{u}}_{h}\left(t^{\left(n_{i}\right)}, \theta^{k}\right)-\underline{\mathbf{u}}_{h}^{p}\left(t^{\left(n_{i}\right)}, \theta^{k}\right)\right\|_{\mathcal{R}^{\mathcal{N}_{d}}} \\
& +\sum_{k=1}^{\mathcal{N}_{p}} \sum_{i=1}^{\mathcal{N}_{t}}\left\|\underline{\mathbf{u}}_{h}^{p}\left(t^{\left(n_{i}\right)}, \theta^{k}\right)-\underline{\mathbf{u}}_{h}^{r}\left(t^{\left(n_{i}\right)}, \theta^{k}\right)\right\|_{\mathcal{R}^{\mathcal{N}_{d}}} \\
\leq & \rho_{t}^{\frac{1}{2}} \mathcal{M}_{1}+\rho_{\theta}^{\frac{1}{2}} \mathcal{M}_{2} \\
& +\left(\mathcal{N}_{t p}\right)^{\frac{1}{2}}\left(\sum_{j=1}^{L_{\mathbf{u}}}\left(\rho_{j, t o l}^{2} \sum_{i=1}^{r_{\mathbf{u}, q}^{j}}\left(\delta_{\mathbf{u}, i}^{j}\right)^{2}\right)\right)^{\frac{1}{2}}, \mathbf{u} \in\{\mathbf{E}, \mathbf{H}\} .
\end{aligned}
$$

In particular, (31) can be used to assess the truncation errors due to the POD method for constructing the RB functions, and the SVD method for the training data of projection coefficients.

Remark 6. The main computational cost of CSI-based approximation is to compute two linear systems via chasing method, and the complexity of which is $\mathcal{O}\left(\mathcal{N}_{t}\right)+\mathcal{O}\left(\mathcal{N}_{p}\right)$ for the $i$-th $(i=$ $\left.1,2, \cdots, l_{\mathbf{u}}^{j}\right)$ discrete time- and parameter-modes of $j$-th $\left(j=1,2, \cdots, L_{\mathbf{u}}\right)$ projection coefficient. So, the total complexity is $\left(\mathcal{O}\left(\mathcal{N}_{t}\right)+\mathcal{O}\left(\mathcal{N}_{p}\right)\right) \times \sum_{j=1}^{L_{\mathbf{u}}} l_{\mathbf{u}}^{j}$. Besides, computing the $S V D$ of an $\mathcal{N}_{t} \times \mathcal{N}_{p}$ matrix $\mathbf{Q}_{\mathbf{u}}^{j}$ has complexity $O\left(\mathcal{N}_{t} \mathcal{N}_{p} \times \max \left(\mathcal{N}_{t}, \mathcal{N}_{p}\right)\right)$, so the total complexity is $O\left(\mathcal{N}_{t} \mathcal{N}_{p} \times\right.$ $\left.\max \left(\mathcal{N}_{t}, \mathcal{N}_{p}\right)\right) \times L_{\mathbf{u}}$, which is always inexpensive.

Remark 7. As an inherent drawback of the interpolation-based non-intrusive $R B$ method, the POD-CSI method cannot guarantee the extrapolation results at the time/parameter values outside the coverage of training data. Some regression approaches may be considered to address this issue, such as autoregression [53], and residual-based ANNs ( $R$-ANNs) regression [35], where the regression models are used to approximate the mappings from an input vector $\left(t, \theta, \alpha_{\mathbf{u}}(t, \theta)\right)$ of dimension $\operatorname{dim}(\mathcal{P})+1+L_{\mathbf{u}}=d+1+L_{\mathbf{u}}$ to an output vector $r_{\mathbf{u}}(t, \theta)$ of dimension $L_{\mathbf{u}}$. However, the accuracy of the predictions must be carefully assessed.

\subsection{A surrogate error model}

It would be useful if ROM could quickly assess an error when predicting a target solution. In the literature $[2,54]$, regression models have been used to approximate the errors introduced by ROM. Based on the similar idea, we use the time/parameter $\left(t^{\left(n_{i}\right)}, \theta^{k}\right)$ in a testing set $\mathcal{T}_{\hbar}^{t e} \times \mathcal{P}_{\hbar}^{t e}$ with $\mathcal{P}_{\hbar}^{t e} \cap \mathcal{P}_{\hbar}^{t r}=\emptyset$ and the corresponding error of reduced-order solution to construct an error surrogate denoted by

$$
\widehat{e}_{\mathbf{u}, \mathrm{POD}-\mathrm{CSI}}(t, \theta): \mathcal{T} \times \mathcal{P} \rightarrow \mathcal{R}, \quad \mathbf{u} \in\{\mathbf{E}, \mathbf{H}\},
$$

which is constructed from the following training dataset via the CSI method

$$
\mathcal{E}_{\mathbf{u}, \hbar}^{t e}=\left\{e_{\mathbf{u}, \mathrm{POD}-\mathrm{CSI}}\left(t^{\left(n_{i}\right)}, \theta^{k}\right),\left(t^{\left(n_{i}\right)}, \theta^{k}\right) \in \mathcal{T}_{\hbar}^{t e} \times \mathcal{P}_{\hbar}^{t e}, \mathbf{u} \in\{\mathbf{E}, \mathbf{H}\}\right\} .
$$

Here, the testing parameter set $\mathcal{P}_{\hbar}^{t e}$ is generated via randomized latin-hypercube-sampling (LHS), the testing time set $\mathcal{T}_{\hbar}^{t e}$ is randomly chosen at last period of the physical simulation, and $e_{\mathbf{u}, \mathrm{POD}-\mathrm{CSI}}$ is the relative error between the DGTD and POD-CSI solutions defined by

$$
e_{\mathbf{u}, \mathrm{POD}-\mathrm{CSI}}(t, \theta)=\frac{\left\|\underline{\mathbf{u}}_{h}(t, \theta)-\underline{\mathbf{u}}_{h}^{r}(t, \theta)\right\|_{\mathcal{R}^{\mathcal{N}_{d}}}}{\left\|\underline{\mathbf{u}}_{h}(t, \theta)\right\|_{\mathcal{R}^{\mathcal{N}_{d}}}}=\frac{\left\|\underline{\mathbf{u}}_{h}(t, \theta)-\Phi_{\mathbf{u}} \alpha_{\mathbf{u}}(t, \theta)\right\|_{\mathcal{R}^{\mathcal{N}_{d}}}}{\left\|\underline{\mathbf{u}}_{h}(t, \theta)\right\|_{\mathcal{R}^{\mathcal{N}_{d}}}}, \quad \mathbf{u} \in\{\mathbf{E}, \mathbf{H}\} .
$$

In particular, we still rewrite the error dataset $\mathcal{E}_{\mathbf{u}, \hbar}^{t e}$ in matrix form, and use the CSI method to approximate the dominating discrete time and parameter modes of the error matrix, which is similar to (26) to $(29)$. 


\section{Numerical results}

In this section, we present some numerical results by considering the problems of the scattering of plane wave by a dielectric cylinder and a multi-layer heterogeneous medium to evaluate the non-intrusive POD-CSI method. We adopt the transverse magnetic (TM) formulation of the time-domain Maxwell's equations (1), i.e., $\mathbf{E}=\left(0,0, E_{z}\right)^{T}$ and $\mathbf{H}=\left(H_{x}, H_{y}, 0\right)^{T}$. The main objective is to show that, with few HDM evaluations, we can construct a ROM that is capable of computing inexpensively the electromagnetic scattering problems for a range of material properties $\theta \in \mathcal{P}$.

In all scattering scenarios considered here, the excitation is a plane wave such that $E_{z}^{\text {inc }}=$ $\cos (-k x+\omega t)$ and $H_{y}^{\text {inc }}=-\cos (-k x+\omega t)$ with $\omega=2 \pi f$ being the angular frequency of the incident wave frequency $f=300 \mathrm{MHz}$, and $k$ being the wave number in vacuum. In addition to (34), we also define the following relative projection error $e_{\mathbf{u}, \operatorname{Pro}}(t, \theta)$ to evaluate the accuracy of the results at new time-parameter location $(t, \theta)$

$$
e_{\mathbf{u}, \operatorname{Pro}}(t, \theta)=\frac{\left\|\underline{\mathbf{u}}_{h}(t, \theta)-\underline{\mathbf{u}}_{h}^{p}(t, \theta)\right\|_{\mathcal{R}^{\mathcal{N}_{d}}}}{\left\|\underline{\mathbf{u}}_{h}(t, \theta)\right\|_{\mathcal{R}^{\mathcal{N}_{d}}}}=\frac{\left\|\underline{\mathbf{u}}_{h}(t, \theta)-\Phi_{\mathbf{u}} \Phi_{\mathbf{u}}^{T} \underline{\mathbf{u}}_{h}(t, \theta)\right\|_{\mathcal{R}^{\mathcal{N}_{d}}}}{\left\|\underline{\mathbf{u}}_{h}(t, \theta)\right\|_{\mathcal{R}^{\mathcal{N}_{d}}}}, \quad \mathbf{u} \in\{\mathbf{E}, \mathbf{H}\} .
$$

The above errors are evaluted on a testing time/parameter sampling $\mathcal{T}_{\hbar}^{t e} \times \mathcal{P}_{\hbar}^{t e}$ of size $\mathcal{N}_{t e}$. In particular, the average of the relative errors are used to measure the accuracy of the ROM in our numerical experiments, which are defined as

$$
\bar{e}_{\mathbf{u}, \text { Pro }}=\frac{\sum_{(t, \theta) \in \mathcal{T}_{\hbar}^{t e} \times \mathcal{P}_{\hbar}^{t e}} e_{\mathbf{u}, \text { Pro }}(t, \theta)}{\mathcal{N}_{t e}}, \bar{e}_{\mathbf{u}, \text { POD-CSI }}=\frac{\sum_{(t, \theta) \in \mathcal{T}_{\hbar}^{t e} \times \mathcal{P}_{\hbar}^{t e}} e_{\mathbf{u}, \text { POD-CSI }}(t, \theta)}{\mathcal{N}_{t e}}, \mathbf{u} \in\{\mathbf{E}, \mathbf{H}\} .
$$

Besides, we will calculate the RCS which describes the distribution of energy scattered by a target in the path of a radar beam. The RCS is defined as for the incident TM wave

$$
\sigma_{\mathrm{TM}}=\lim _{r \rightarrow \infty} 2 \pi r \frac{\left|\bar{E}_{z}^{\mathrm{sca}}\right|^{2}}{\left|\bar{E}_{z}^{\text {inc }}\right|^{2}}=\lim _{r \rightarrow \infty} 2 \pi r \frac{\left|\overline{\mathbf{H}}^{\mathrm{sca}}\right|^{2}}{\left|\overline{\mathbf{H}}^{\text {inc }}\right|^{2}},
$$

where $\bar{E}_{z}^{\text {sca }}\left(\right.$ resp. $\left.\overline{\mathbf{H}}^{\text {sca }}\right)$ and $\bar{E}_{z}^{\text {inc }}\left(\right.$ resp. $\left.\overline{\mathbf{H}}^{\text {inc }}\right)$ are the frequency-domain scattered and incident electric (resp. magnetic) fields, respectively, and $r$ is the distance from the observation point to the scatterer. The CSI-based approximation of reduced-order coefficient matrices are constructed via the MATLAB functions spline and griddedInterpolant in the first and second numerical experiments, respectively. The DGTD and POD-CSI methods are implemented in MATLAB. All simulations are run on a workstation equipped with an Intel Xeon CPU running at $3.70 \mathrm{GHz}$, and with $32 \mathrm{~GB}$ of RAM memory.

\subsection{Scattering of plane wave by a dielectric cylinder}

We first consider the electromagnetic scattering of plane wave by an infinite dielectric cylinder. The radius of the cylinder is $0.6 \mathrm{~m}$. The cylinder is centered at the origin and the computational domain is artificially truncated by the square $\Omega_{\square}=[-2.6 \mathrm{~m}, 2.6 \mathrm{~m}] \times[-2.6 \mathrm{~m}, 2.6 \mathrm{~m}]$. We impose the first order Silver-Müller ABC on the square delimiting the domain (the distance between the object and the absorbing boundary is $2 \lambda$ with $\lambda$ being the wavelength of the incident wave in the vacuum.). The relative permittivity of the cylinder ranges $\varepsilon_{r} \in[1,5]$, and its relative permeability is set to $\mu_{r}=1$ (nonmagnetic material), i.e., $\mathcal{P}=\left\{\theta: \theta=\varepsilon_{r} \in[1,5]\right\} \subset \mathcal{R}$. The medium exterior to the dielectric cylinder is assumed to be vacuum. All the simulations are performed using a triangular mesh with 2575 nodes and 5044 elements of which 1092 elements are located inside the cylinder. 
The physical simulation time is set to 50 periods (which corresponds to $50 \mathrm{~m}$ in normalized unit) of the incident wave oscillation with time step $\Delta t=0.0037 \mathrm{~m}$ using the DGTD method with a $\mathbb{P}_{2}$ approximation (the interpolation order is assumed to be uniform across all mesh elements). The training parameter samples $\mathcal{P}_{\hbar}^{t r}$ are generated via uniform sampling between 1 and 5 at interval of $\Delta p=1,0.5,0.25,0.1$, and 0.05 each, respectively, resulting in $\mathcal{N}_{p}=5,9,17$, 41 , and 81 points in parameter space. The snapshots are then obtained every time step at last period for each selected parameter point. Here, $\mathcal{T}_{\hbar}^{t r}=\{49.0024,49.006, \cdots, 49.9623,49.966\}$, and the number of time points is $\mathcal{N}_{t}=263$. The total number of snapshots $\mathcal{N}_{t p}$ is large, so we use the two-step POD method to extract the POD basis functions from all the snapshots. In Figure 1 , the convergence histories of $\bar{e}_{\mathbf{u} \text {,Pro }}$ and $\bar{e}_{\mathbf{u} \text {,POD-CSI }}$ on the testing set $\mathcal{T}_{\hbar}^{t e} \times \mathcal{P}_{\hbar}^{t e}$ for different training data are plotted, in which the truncation tolerance $\rho_{t}=1 \times 10^{-1}$, the size of the testing parameter set $\mathcal{P}_{\hbar}^{t e}$ is 40 , and the testing time set $\mathcal{T}_{\hbar}^{t e}=\mathcal{T}_{\hbar}^{t r}$. As can be seen, the

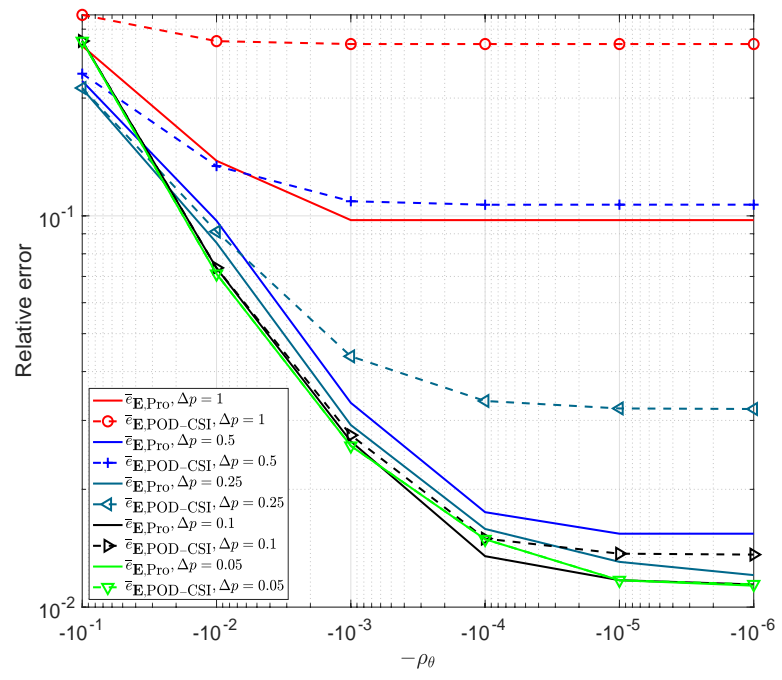

(a)

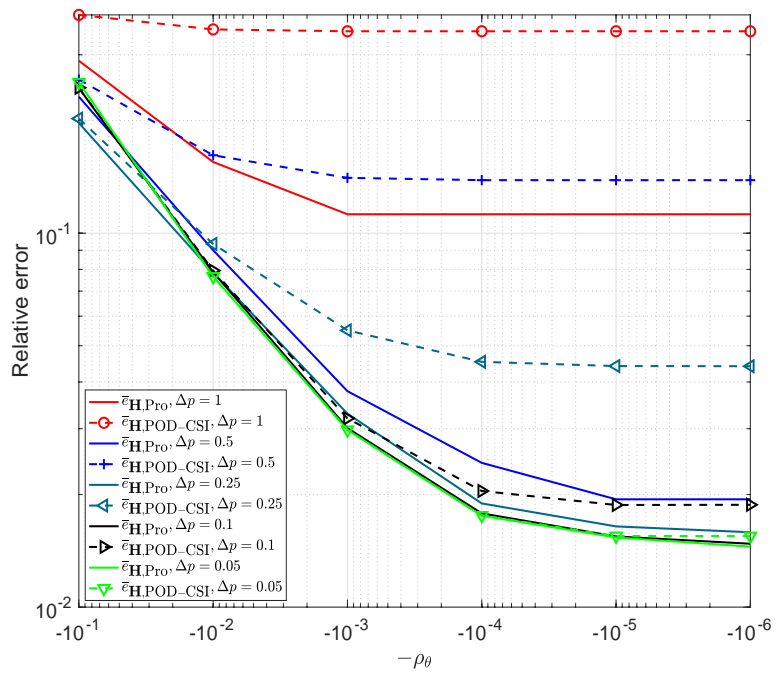

(b)

Figure 1: Scattering of plane wave by a dielectric cylinder: the convergence histories of $\bar{e}_{\mathbf{E}, \text { Pro }}$, and $\bar{e}_{\mathbf{E}, \text { POD-CSI }}$ (a), $\bar{e}_{\mathbf{H}, \text { Pro }}$, and $\bar{e}_{\mathbf{H}, \text { POD-CSI }}$ (b) on the testing set with the truncation tolerances $\rho_{\theta}$.

performance of the POD-CSI method depends on the size of training set and the truncation tolerance $\rho_{\theta}$ used. In other words, the POD-CSI method is effective when large training points and small truncation tolerance $\rho_{\theta}$ are provided. So, we choose $\mathcal{P}_{\hbar}^{t r}=\{1,1.05,1.1, \cdots, 4.95,5\}$ in the following study. The details for the training and testing datasets are listed in Table 2. More-

Table 2: Scattering of plane wave by a dielectric cylinder: settings for the training, and testing datasets.

\begin{tabular}{ccc}
\hline Data set & Training & Testing \\
\hline Parameter sample points & 81, uniform & 40, random (LHS method) \\
Time sample points & 263, uniform & 263 , uniform \\
Size & 21303 & 10520 \\
\hline
\end{tabular}

over, the effect of $\left(\rho_{t}, \rho_{\theta}\right)$ on the average projection and POD-CSI errors $\bar{e}_{\mathbf{u}, P r o}$ and $\bar{e}_{\mathbf{u}, \text { POD-CSI }}$ is investigated to provide guidance on the choice of truncation tolerances, which is shown in Figure 2. We observe that $\bar{e}_{\mathbf{u}, \operatorname{Pro}}\left(\rho_{t}, \rho_{\theta}\right)$ and $\bar{e}_{\mathbf{u}, \text { POD-CSI }}(\mathbf{u} \in\{\mathbf{E}, \mathbf{H}\})$ are monotonically increasing as $\rho_{\theta}$ increases from $1 \times 10^{-5}$ to $1 \times 10^{-1}$ for the fixed error bound $\rho_{t}$. In addition, one can find that $\bar{e}_{\mathbf{E}, \text { POD-CSI }}$ for $\rho_{t}$ from $1 \times 10^{-1}$ to $1 \times 10^{-3}$ is smaller than that from $1 \times 10^{-4}$ to $1 \times 10^{-6}$ when the error bound $\rho_{\theta}$ is reduced from $1 \times 10^{-5}$ to $1 \times 10^{-6}$, and $\bar{e}_{\mathbf{H}, \text { POD-CSI }}$ has similar feature when the error bound $\rho_{\theta}$ is reduced from $1 \times 10^{-4}$ to $1 \times 10^{-6}$. In particular, 
$\bar{e}_{\mathbf{u}, \text { Pro }}\left(\rho_{t}, \rho_{\theta}\right)$ and $\bar{e}_{\mathbf{u}, \text { POD-CSI }}(\mathbf{u} \in\{\mathbf{E}, \mathbf{H}\})$ are basically unchanged when the error bound $\rho_{t}$ is reduced from $1 \times 10^{-1}$ to $1 \times 10^{-3}$ for the fixed error bound $\rho_{\theta}$, i.e., the small dimension POD basis functions can capture the information of the high fidelity DGTD solutions for each parameter. Combining Figure 2 (a) and (b), we select the error bounds $\rho_{t}=1 \times 10^{-1}$ and

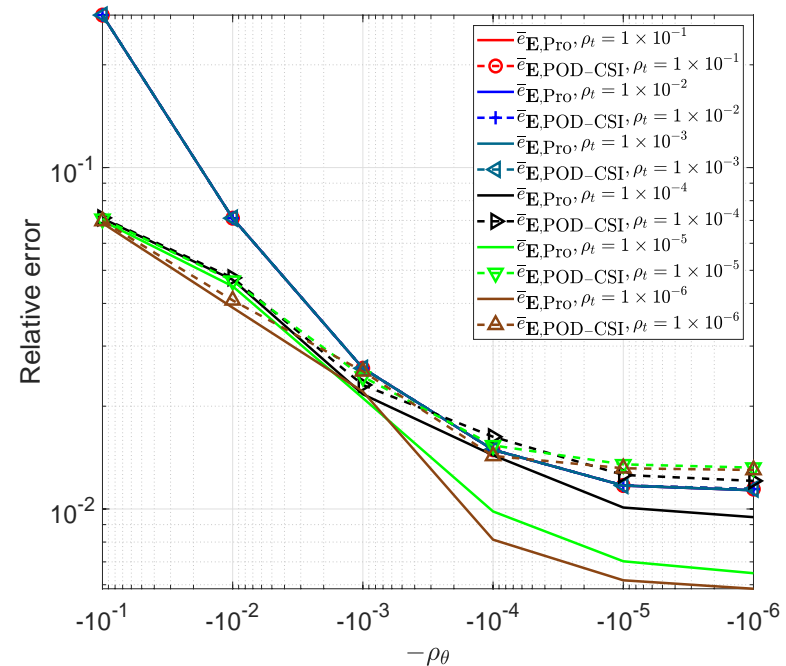

(a)

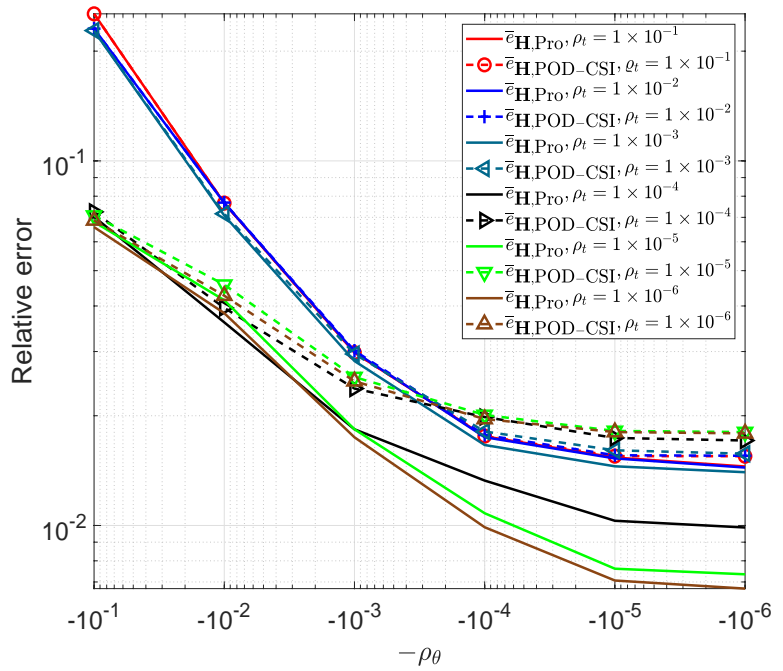

(b)

Figure 2: Scattering of plane wave by a dielectric cylinder: the contour of projection and POD-CSI errors $\bar{e}_{\mathbf{E}, \text { Pro }}$ and $\bar{e}_{\mathbf{E}, \text { POD-CSI }}(\mathrm{a}), \bar{e}_{\mathbf{H}, \text { Pro }}$ and $\bar{e}_{\mathbf{H}, \text { POD-CSI }}$ (b) based on the two-step POD method with the truncation tolerances $\left(\rho_{t}, \rho_{\theta}\right)$.

$\rho_{\theta}=1 \times 10^{-5}$ in the two-step POD method, which results in a set of $L_{E_{z}}=22, L_{H_{x}}=20$, and $L_{H_{y}}=35$ POD basis functions. The corresponding projection and POD-CSI errors are shown in Table 3. In particular, we can find that the dimension $L_{H_{x}}$ is relatively large. Because

Table 3: Scattering of plane wave by a dielectric cylinder: the average projection and POD-CSI errors on the testing set.

\begin{tabular}{ccccc}
\hline Average relative error & $\bar{e}_{\mathbf{E}, \text { Pro }}$ & $\bar{e}_{\mathbf{E}, \text { POD-CSI }}$ & $\bar{e}_{\mathbf{H}, \text { Pro }}$ & $\bar{e}_{\mathbf{H}, \text { POD-CSI }}$ \\
\hline Value & $1.171 \times 10^{-2}$ & $1.172 \times 10^{-2}$ & $1.535 \times 10^{-2}$ & $1.549 \times 10^{-2}$ \\
\hline
\end{tabular}

when the relative permittivity is close to 1 , the numerical solutions of $H_{x}$ are basically errors oscillating around 0 , there is a lot of noise information in the high fidelity solutions of $H_{x}$. So more POD basis functions are needed to capture the characteristics of $H_{x}$.

The POD-CSI models are constructed as the combination of time- and parameter-modes obtained by the CSI method, some of which are shown in Figures 3 and 4 . In particular, the SVD truncation criterion for all projection coefficient matrices are set to $\rho_{l, \text { tol }}=1 \times 10^{-4}$ for $1 \leq l \leq 5, \rho_{l, \text { tol }}=5 \times 10^{-4}$ for $6 \leq l \leq 10, \rho_{l, \text { tol }}=1 \times 10^{-3}$ for $11 \leq l \leq 20$, and $\rho_{l, \text { tol }}=5 \times 10^{-3}$ for $21 \leq l \leq L_{\mathbf{u}}(\mathbf{u}=\mathbf{E}, \mathbf{H})$. The smaller criterions are defined for the preceding coefficients since they play a dominant role in the accuracy of the reduced-order solutions. In particular, the POD-CSI models can be stored in the offline phase, and for new parameters we can directly call these models to calculate the electromagnetic fields in the online stage instead of runing DGTD method. The POD-CSI models can be finished within minutes.

After the offline stage, in order to assess the accuracy of POD-CSI method, the online tests are performed on the solutions for testing parameters, and chosen as $\theta^{1}=2.215, \theta^{2}=3.215$ and $\theta^{3}=4.215$. The 1 - $\mathrm{D}$ x-wise distribution of the real part of $H_{y}$ in the Fourier domain 
The 1th coefficent - time modes

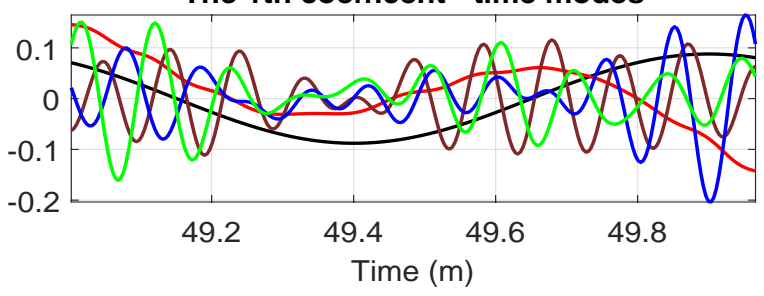

The 5th coefficent - time modes

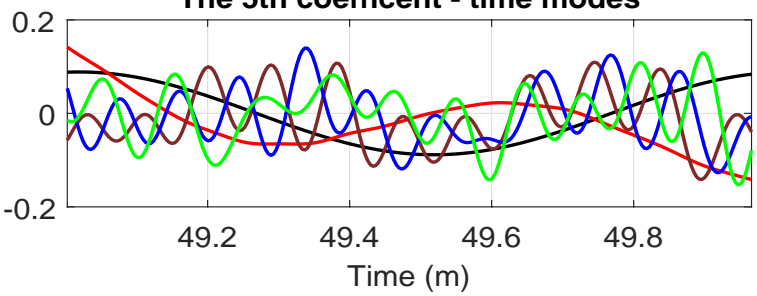

Time $(\mathrm{m})$

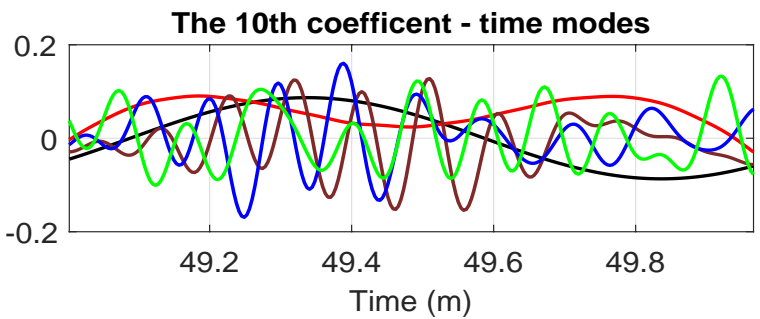

The 1th coefficent - parameter modes

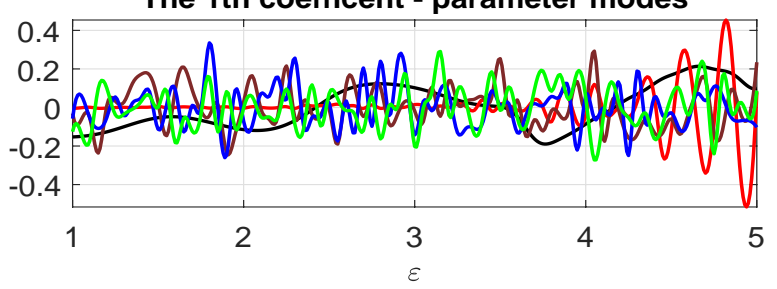

The 5th coefficent - parameter modes
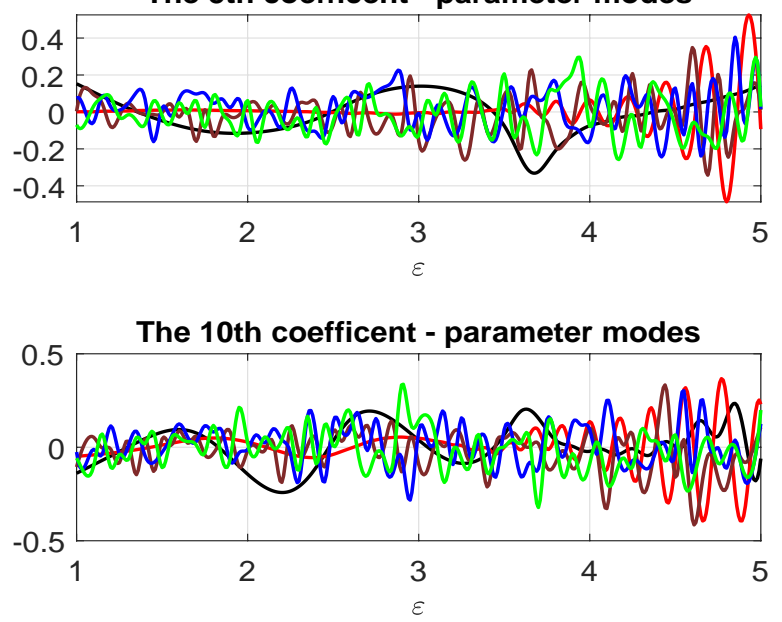

Figure 3: Scattering of plane wave by a dielectric cylinder: the 1th, 5th, and 10th time (left) and parameter modes (right) for $E_{z}$ (the 2 th mode: black, the 4 th mode: red, the 6 th mode: brown; the 8 th mode: blue; the 10th mode: green).

over the last period of simulation along $y=0$ is displayed in Figure 5 (a),(c),(e). With this single frequency calculation, the backscatter and bistatic RCS is computed. The bistatic RCS is shown in Figure 5 (b),(d),(f). We find that the DGTD and reduced-order solutions are matching well with each other, ensuring the effectiveness of the proposed method. The time evolution of the relative projection error $e_{\mathbf{u}, \text { Pro }}$, the POD-CSI error $e_{\mathbf{u}, \text { POD-CSI }}$ and the surrogate error $\widehat{e}_{\mathbf{u}, \text { POD-CSI }}(\mathbf{u} \in\{\mathbf{E}, \mathbf{H}\})$ for the testing parameters $\theta^{1}, \theta^{2}$, and $\theta^{3}$ are shown in Figure 6 , where the size of the parameter sampling $\mathcal{P}_{\hbar}^{t e}$ is 20 . We observe that the error of POD-CSI method is mainly the projection error from Figure 6 . In addition, as surrogates for relative errors, the CSI method provide non-rigorous but practical error indicators for the reduced-order solutions. The accuracy of the surrogate error model can be improved by increasing the error training points. The correspoinding max errors are listed in Table 4.

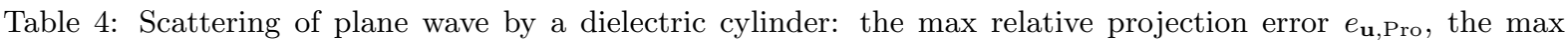
POD-CSI error $e_{\mathbf{u}, \text { POD-CSI }}$ and the max surrogate error $\widehat{e}_{\mathbf{u}, \text { POD-CSI }}(\mathbf{u}=\mathbf{E}, \mathbf{H})$ for the testing parameters $\theta^{1}, \theta^{2}$, and $\theta^{3}$.

\begin{tabular}{ccccccc}
\hline Test parameters & $e_{\mathbf{E}, \text { Pro }}$ & $e_{\mathbf{E}, \text { POD-CSI }}$ & $\widehat{e}_{\mathbf{E}, \text { POD-CSI }}$ & $e_{\mathbf{H}, \text { Pro }}$ & $e_{\mathbf{H}, \text { POD-CSI }}$ & $\widehat{e}_{\mathbf{H}, \text { POD-CSI }}$ \\
\hline$\theta^{1}$ & $1.246 \times 10^{-2}$ & $1.246 \times 10^{-2}$ & $1.218 \times 10^{-2}$ & $1.542 \times 10^{-2}$ & $1.544 \times 10^{-2}$ & $1.557 \times 10^{-2}$ \\
$\theta^{2}$ & $1.375 \times 10^{-2}$ & $1.376 \times 10^{-2}$ & $1.349 \times 10^{-2}$ & $1.796 \times 10^{-2}$ & $1.821 \times 10^{-2}$ & $1.812 \times 10^{-2}$ \\
$\theta^{3}$ & $1.607 \times 10^{-2}$ & $1.608 \times 10^{-2}$ & $1.535 \times 10^{-2}$ & $2.532 \times 10^{-2}$ & $2.548 \times 10^{-2}$ & $2.413 \times 10^{-2}$ \\
\hline
\end{tabular}

A performance comparison between the POD-CSI (offline cost and online cost) and DGTD methods is given in Table 5. We find that most of the offline cost are due to the generation of the high fidelity solutions from Table 5. In particular, the online cost of the POD-CSI 

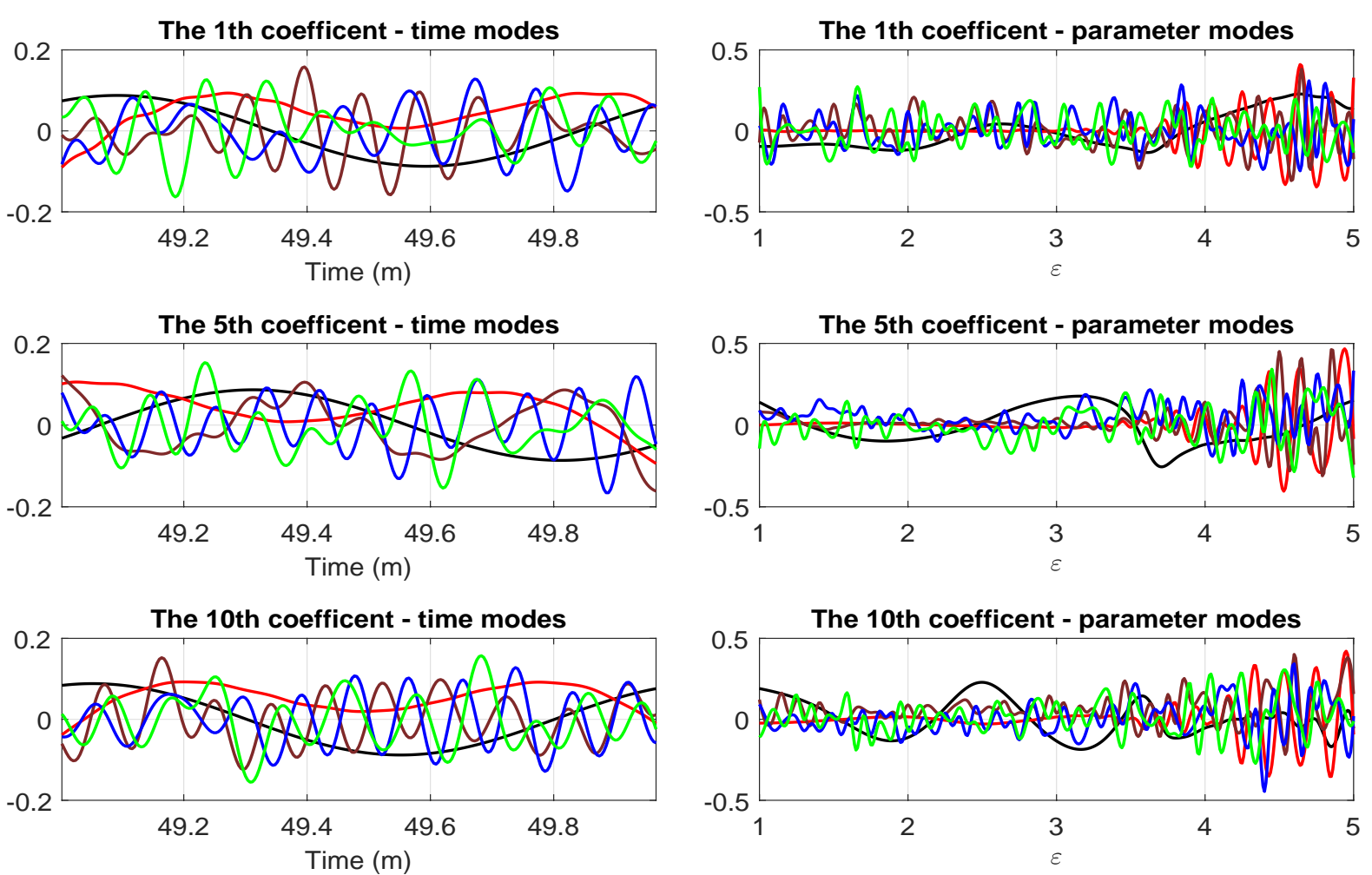

Figure 4: Scattering of plane wave by a dielectric cylinder: the 1th, 5 th, and 10th time (left) and parameter modes (right) for $H_{y}$ (the 2 th mode: black, the 4th mode: red, the 6th mode: brown; the 8th mode: blue; the 10th mode: green).

method for a new parameter is extremely small compared to the DGTD method. For this scattering problem, we can conclude that the non-intrusive POD-CSI method is effective for the parameterized time-domain Maxwell's equations.

Table 5: Scattering of plane wave by a dielectric cylinder: the comparison between the POD-CSI (offline and online) and DGTD methods in terms of CPU time. The unit of time cost is second.

\begin{tabular}{ccccc}
\hline \multicolumn{3}{c}{ Offline } & \multicolumn{3}{c}{ Online } \\
\hline Snapshots & Two-step POD & CSI & POD-CSI & DGTD \\
$3.948 \times 10^{4}$ & $3.858 \times 10^{1}$ & $1.179 \times 10^{2}$ & 1.363 & $4.874 \times 10^{2}$ \\
\hline
\end{tabular}

\subsection{Scattering of plane wave by a multi-layer heterogeneous medium}

We now study the performance of POD-CSI method in the case of the scattering of plane wave by a multi-layer medium [55]. The computational domain is artificially truncated by a square with $6.4 \mathrm{~m}$ side length on which the first order Silver-Müller ABC is applied. Simulations are performed using a mesh with 2049 nodes and 4016 elements of which 865 elements are located inside the multi-layer medium, where the minimum and maximum mesh edge length is $2.820 \times 10^{-2} \mathrm{~m}$ and $3.513 \times 10^{-1} \mathrm{~m}$, respectively. Table 6 summarizes the distribution and range of material parameters considered for this study.

The physical simulation time is again set to 50 periods of the incident wave oscillation for each 4-dimensional parameter $\theta \in \mathcal{P}$ with time step $\Delta t=0.0038 \mathrm{~m}$ using the DGTD method with a $\mathbb{P}_{2}$ approximation, where $\mathcal{P}=\mathcal{P}^{1} \times \mathcal{P}^{2} \times \mathcal{P}^{3} \times \mathcal{P}^{4} \subset \mathcal{R}^{4}$ with $\varepsilon_{r}^{(i)} \in \mathcal{P}^{i}$ 


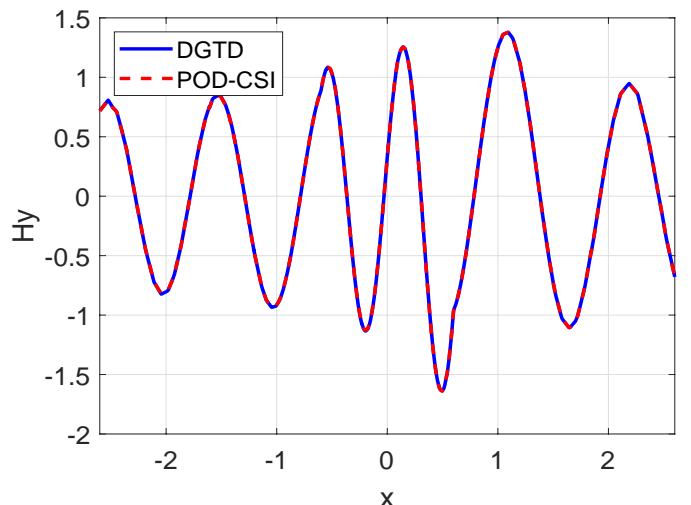

(a)

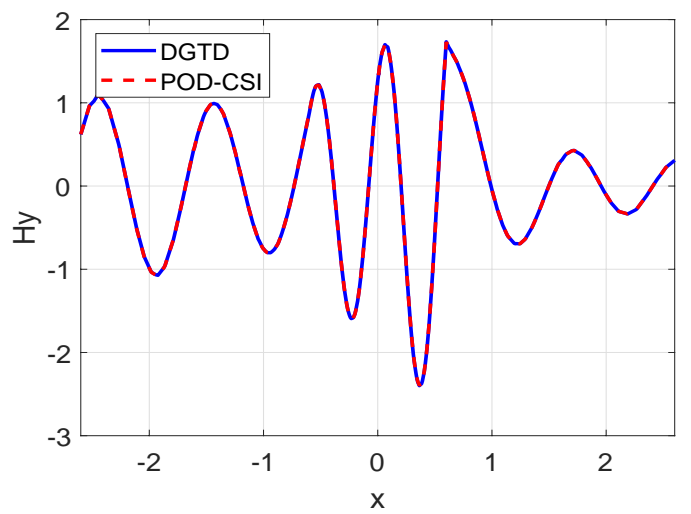

(c)

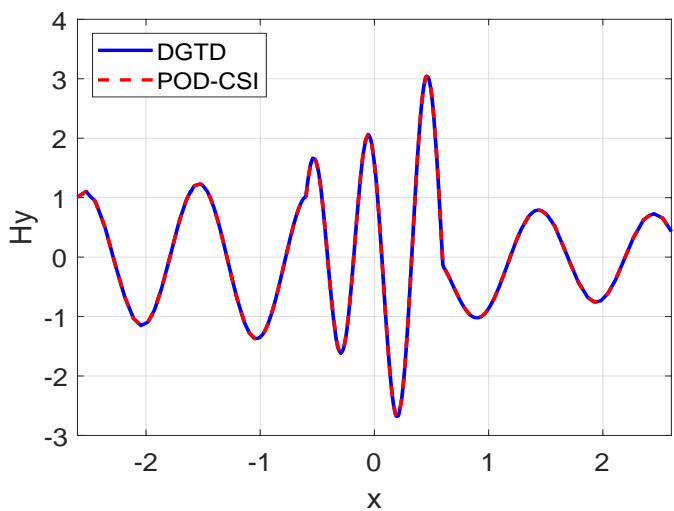

(e)

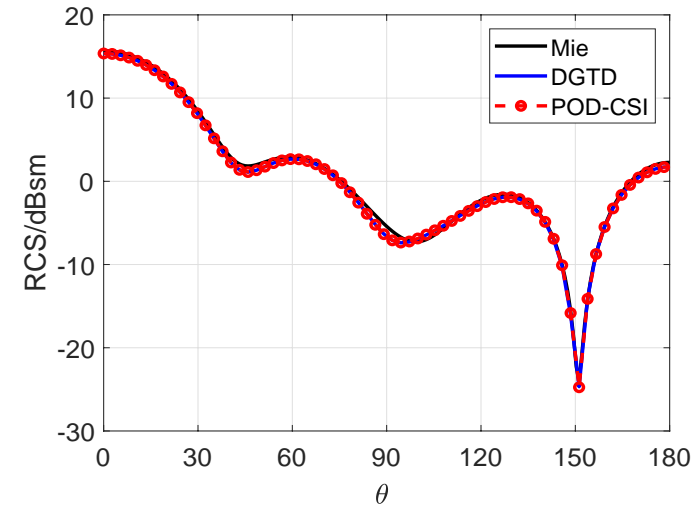

(b)

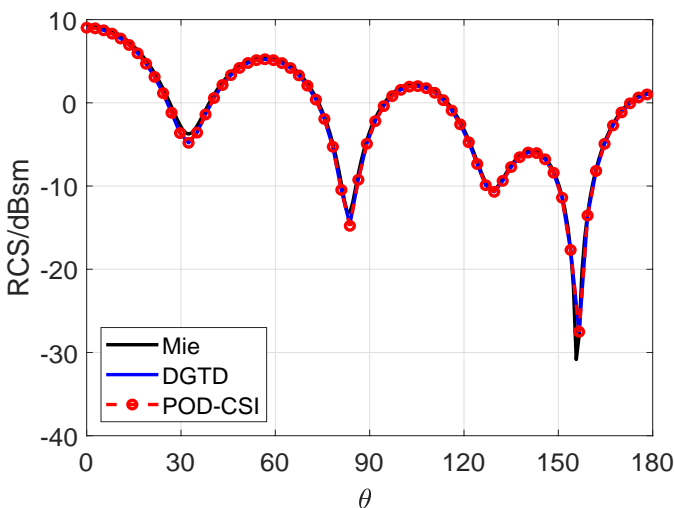

(d)

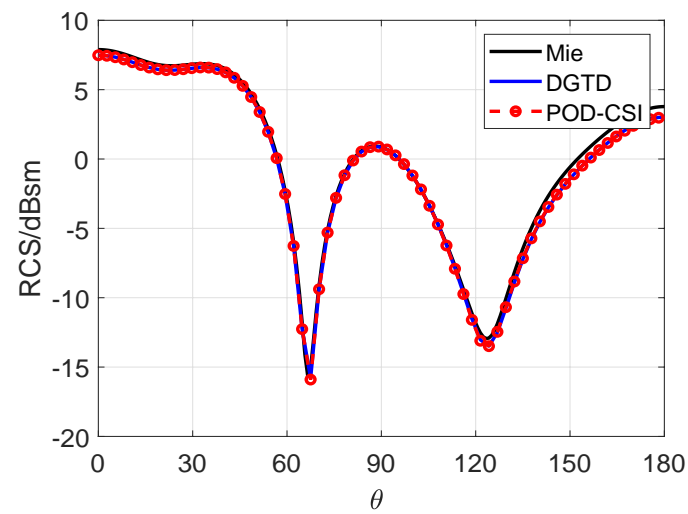

(f)

Figure 5: Scattering of plane wave by a dielectric cylinder: 1-D plots of the real part of DGTD and POD-CSI solutions (DFT) of $H_{y}$ along $y=0$ (left), and bistatic RCS based on Mie series, DGTD and POD-CSI solutions (right) with the testing parameters $\theta^{1}=2.215$ (a)-(b), $\theta^{2}=3.215$ (c)-(d), and $\theta^{3}=4.215$ (e)-(f).

Table 6: Scattering of plane wave by a multi-layer heterogeneous medium: the distribution and range of material parameters.

\begin{tabular}{cccc}
\hline Layer $i$ & $\mathcal{P}^{i}$ & $\mu_{r}^{(i)}$ & $r_{i}$ \\
\hline 1 & $\varepsilon_{r}^{(1)} \in[5.0,5.6]$ & 1 & 0.15 \\
2 & $\varepsilon_{r}^{(2)} \in[3.25,3.75]$ & 1 & 0.3 \\
3 & $\varepsilon_{r}^{(3)} \in[2.0,2.5]$ & 1 & 0.45 \\
4 & $\varepsilon_{r}^{(4)} \in[1.25,1.75]$ & 1 & 0.6 \\
\hline
\end{tabular}



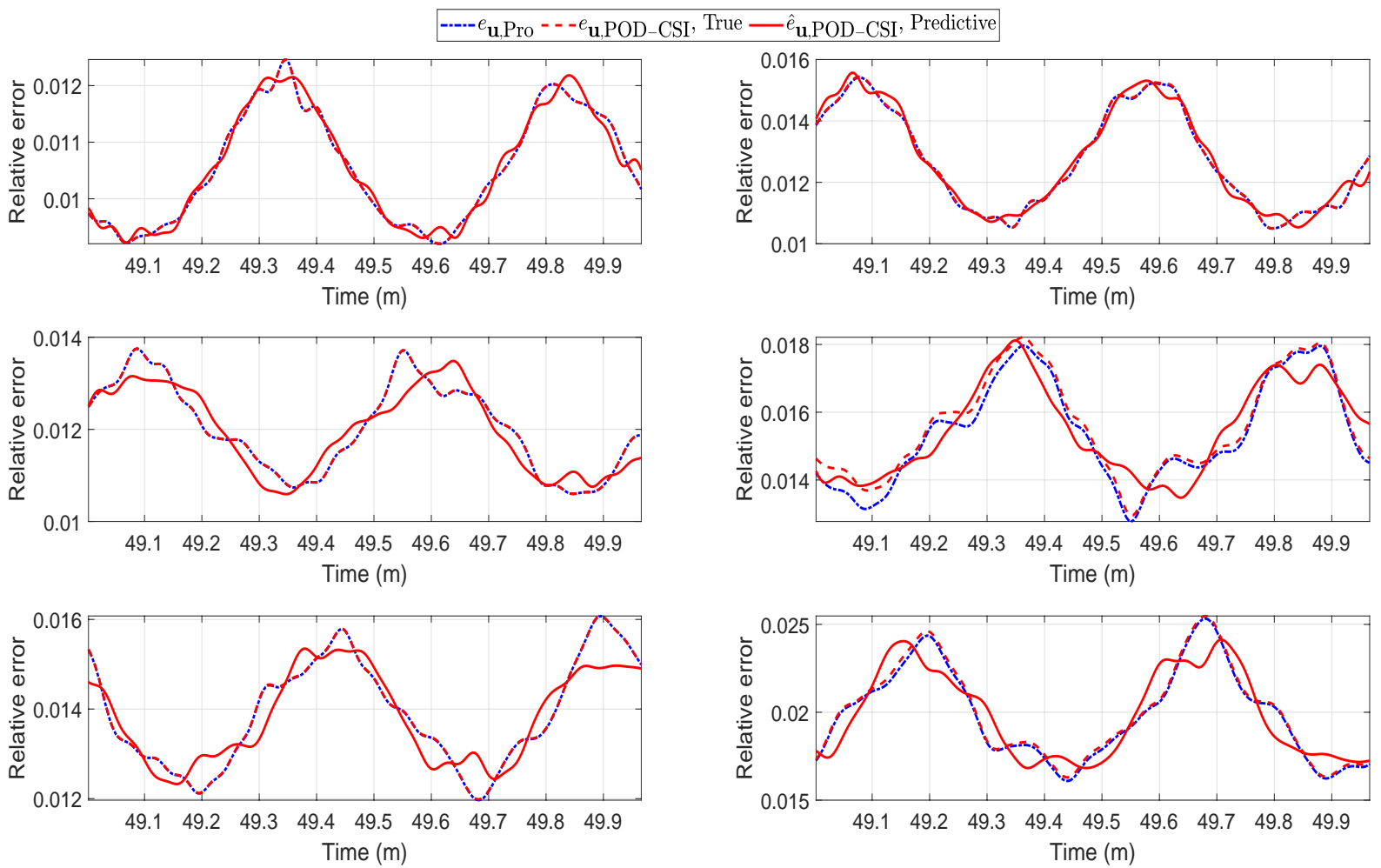

Figure 6: Scattering of plane wave by a dielectric cylinder: the time evolution of the relative projection error $e_{\mathbf{u}, \text { Pro }}$, the POD-CSI error $e_{\mathbf{u}, \text { POD-CSI }}$ and the surrogate error $\widehat{e}_{\mathbf{u}, \text { POD-CSI }}$ for $\mathbf{E}$ (left) and $\mathbf{H}$ (right) with the testing parameters $\theta^{1}=2.215$ (top), $\theta^{2}=3.215$ (middle), and $\theta^{1}=4.215$ (bottom).

$(i=1,2, \cdots, 4)$. The medium exterior to the dielectric cylinder is also assumed to be vacuum. We use a grid sampling of tensor product with $\mathcal{N}_{\Delta p}=2$ and 3 uniform points for each parameter to form the training parameter samples $\mathcal{P}_{\hbar}^{t r}$, respectively, resulting in $\mathcal{N}_{p}=16$ and 81 points. Each choice of the parameter is sampled for $\mathcal{N}_{t}=253$ snapshots in time at last period, i,e., $\mathcal{T}_{\hbar}^{t r}=\{49.0002,49.0041, \cdots, 49.9630,49.9669\}$. We also use the two-step POD method to extract the POD basis functions from all the snapshots. A quantitative evidence of the efficacy of POD-CIS method is shown in Figure 7, which reports the convergence history of $\bar{e}_{\mathbf{u}, \text { Pro }}$ and $\bar{e}_{\mathbf{u}, \text { POD-CSI }}$ on the testing set $\mathcal{T}_{\hbar}^{t e} \times \mathcal{P}_{\hbar}^{t e}$ for different training dataset. As usual, the testing parameter set $\mathcal{P}_{\hbar}^{t e}$ is sampled by a grid sampling of tensor product with 2 points (LHS method) for each parameter, the truncation tolerance $\rho_{t}=1 \times 10^{-1}$, and the testing time set $\mathcal{T}_{\hbar}^{t e}=\mathcal{T}_{\hbar}^{t r}$. We can obtain a conclusion similar to Figure 1 . In the following study, we choose 3 uniform points for each parameter to form the training parameter set, i.e., $\mathcal{P}_{\hbar}^{t r}=\{(5,3.25,2,1.25),(5.3,3.25,2,1.25), \cdots,(5.6,3.75,2.5,1.75)\}$. The details for the training and testing data are summarized in Table 7 . The contours of the truncation tolerance $\left(\rho_{t}, \rho_{\theta}\right)$

Table 7: Scattering of plane wave by a multi-layer heterogeneous medium: settings for the training, and testing datasets.

\begin{tabular}{ccc}
\hline Data set & Training & Testing \\
\hline Parameter sample points & 3, uniform for $\varepsilon_{r}^{(i)}(i=1,2, \cdots, 4)$ & 2, random for $\varepsilon_{r}^{(i)}(i=1,2, \cdots, 4)($ LHS method $)$ \\
Time sample points & 253, uniform & 253, uniform \\
Size & 20493 & 4048 \\
\hline
\end{tabular}




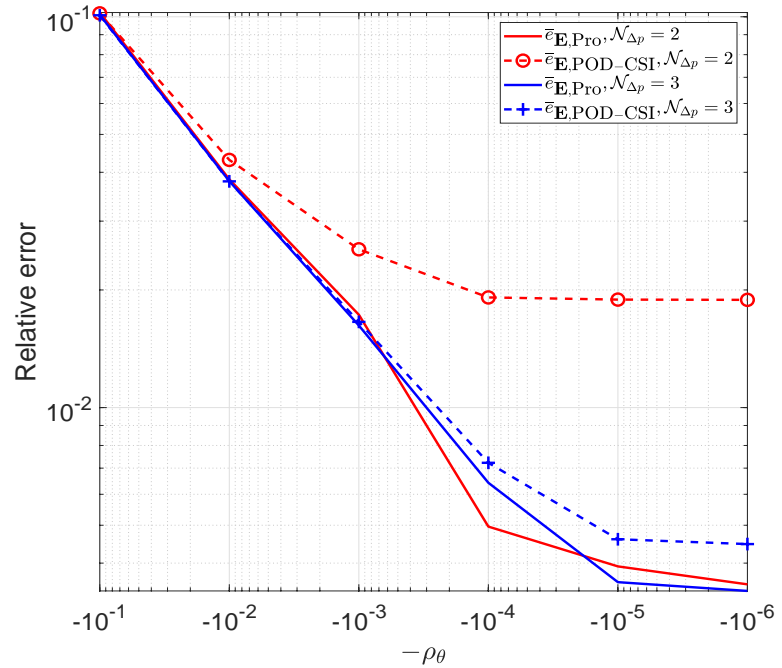

(a)

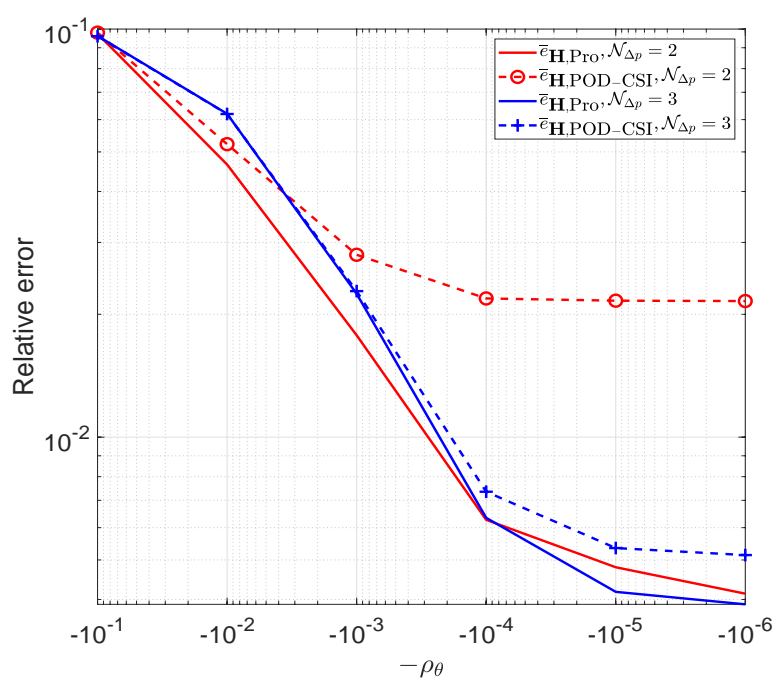

(b)

Figure 7: Scattering of plane wave by a multi-layer heterogeneous medium: the convergence histories of $\bar{e}_{\mathbf{E}, \text { Pro }}$, and $\bar{e}_{\mathbf{E}, \text { POD-CSI }}(\mathrm{a}), \bar{e}_{\mathbf{H}, \text { Pro }}$, and $\bar{e}_{\mathbf{H}, \text { POD-CSI }}(\mathrm{b})$ on the testing set with the truncation tolerances $\rho_{\theta}$.

on the average projection and POD-CSI errors $\bar{e}_{\mathbf{u}, \text { Pro }}$ and $\bar{e}_{\mathbf{u}, \text { POD-CSI }}$ are shown in Figure 8 . As

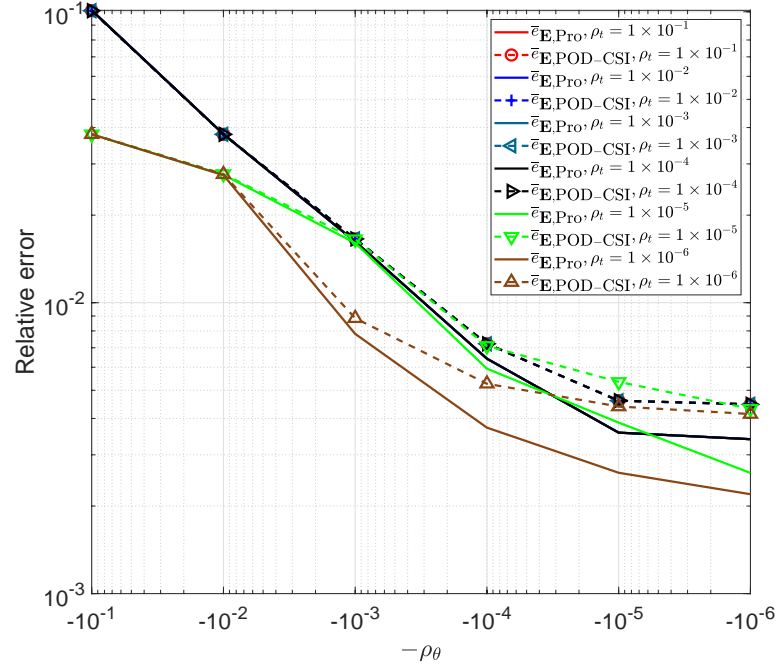

(a)

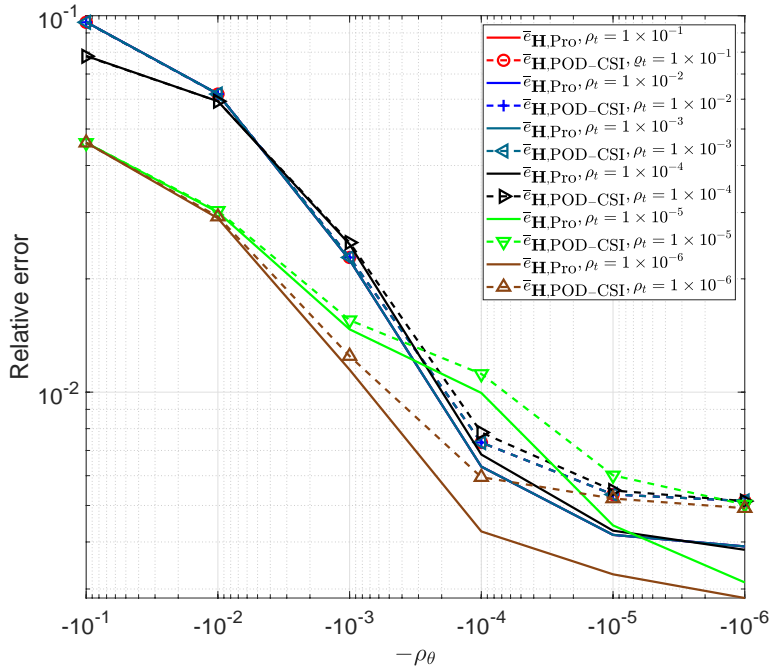

(b)

Figure 8: Scattering of plane wave by a multi-layer heterogeneous medium: the contour of projection and PODCSI errors $\bar{e}_{\mathbf{E} \text {,Pro }}$ and $\bar{e}_{\mathbf{E}, \text { POD-CSI }}(\mathrm{a}), \bar{e}_{\mathbf{H}, \text { Pro }}$ and $\bar{e}_{\mathbf{H}, \text { POD-CSI }}$ (b) based on the two-step POD method with the truncation tolerances $\left(\rho_{t}, \rho_{\theta}\right)$.

shown in the figure, when the error bound $\rho_{\theta}$ is reduced from $1 \times 10^{-5}$ to $1 \times 10^{-6}$, the PODCSI error $\bar{e}_{\mathbf{u}, \text { POD-CSI }}(\mathbf{u} \in\{\mathbf{E}, \mathbf{H}\})$ is basically the same except that $\rho_{t}=1 \times 10^{-5}$. Moreover, $\bar{e}_{\mathbf{u}, \text { Pro }}\left(\rho_{t}, \rho_{\theta}\right)$ and $\bar{e}_{\mathbf{u}, \text { POD-CSI }}(\mathbf{u} \in\{\mathbf{E}, \mathbf{H}\})$ are also monotonically increasing as $\rho_{\theta}$ increases from $1 \times 10^{-5}$ to $1 \times 10^{-1}$ for the fixed error bound $\rho_{t}$. So, the truncation errors in the two-step POD are chosen $\rho_{t}=1 \times 10^{-1}$ and $\rho_{\theta}=1 \times 10^{-5}$ combining Figure 8 (a) and (b), which results in a set of $L_{E_{z}}=15, L_{H_{x}}=17$, and $L_{H_{y}}=15$ orthogonal modes. For comparison we have the DoF of DGTD method $\mathcal{N}_{d}=37236$. The corresponding average projection and POD-CSI errors on the testing set are presented in Table 8 .

After the SVD of all projection coefficients matrices, the POD-CSI models are constructed as the combination of time- and parameter-modes obtained by CSI method, and some of the time- 
Table 8: Scattering of plane wave by a dielectric cylinder: the average projection and POD-CSI errors on the testing set.

\begin{tabular}{ccccc}
\hline Average relative error & $\bar{e}_{\mathbf{E}, \text { Pro }}$ & $\bar{e}_{\mathbf{E}, \text { POD-CSI }}$ & $\bar{e}_{\mathbf{H}, \text { Pro }}$ & $\bar{e}_{\mathbf{H}, \text { POD-CSI }}$ \\
\hline Value & $3.576 \times 10^{-3}$ & $4.604 \times 10^{-3}$ & $4.177 \times 10^{-3}$ & $5.348 \times 10^{-3}$ \\
\hline
\end{tabular}

models for $E_{z}$ and $H_{y}$ are shown in Figure 9. Similar to the section 5.1, the different truncation criterions $\rho_{l \text {,tol }}=5 \times 10^{-5}$ for $1 \leq l \leq 5, \rho_{l, \text { tol }}=1 \times 10^{-4}$ for $6 \leq l \leq 10, \rho_{l, \text { tol }}=5 \times 10^{-4}$ for $11 \leq l \leq 20$ and $\rho_{l, \text { tol }}=1 \times 10^{-3}$ for $21 \leq l \leq L_{\mathbf{u}}(\mathbf{u}=\mathbf{E}, \mathbf{H})$ are adopted for the SVD of the projection coefficient matrices.
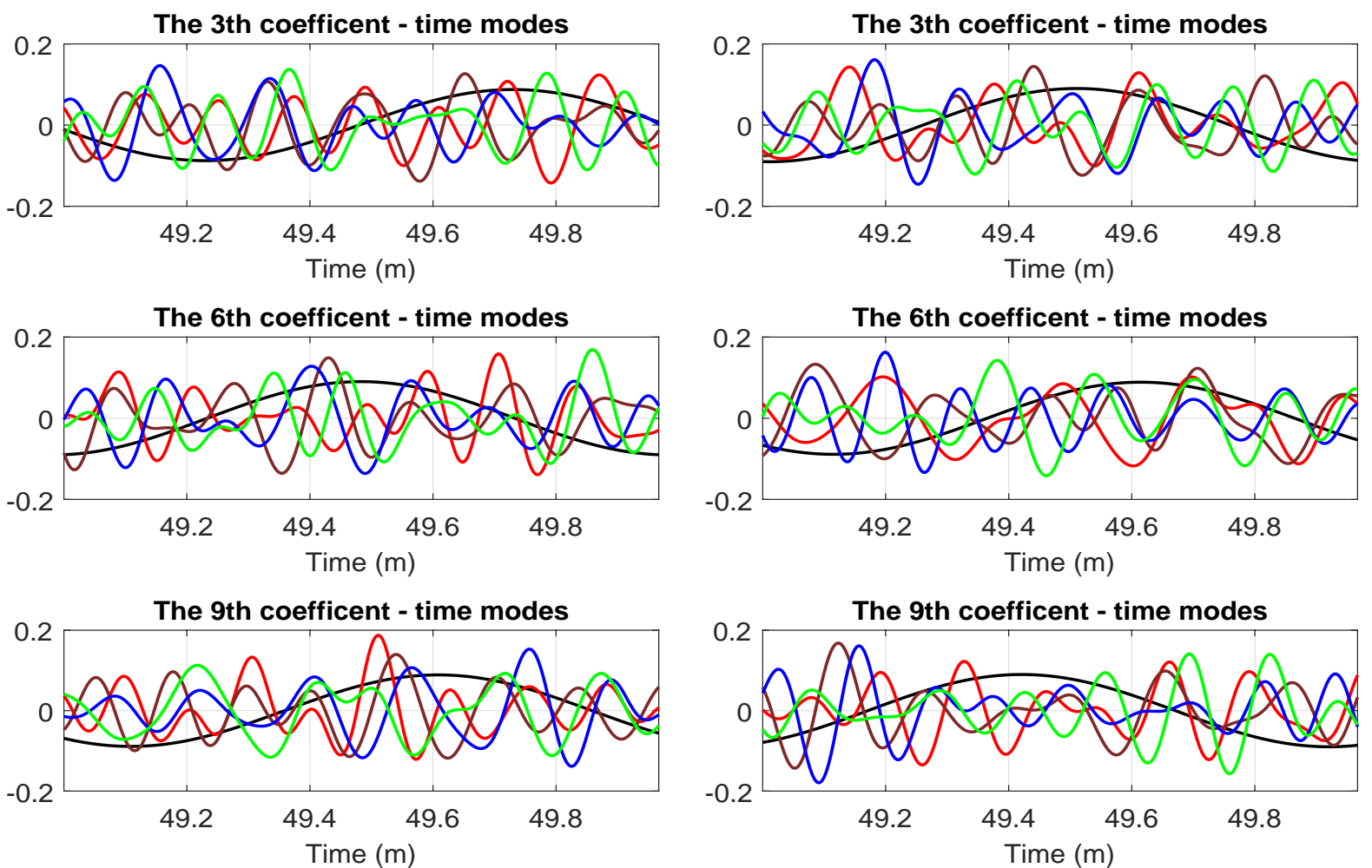

Figure 9: Scattering of plane wave by a multi-layer heterogeneous medium: the 3th, 6 th, and 9th time modes for $E_{z}$ (left) and $H_{y}$ (right) (the 2th mode: black, the 4th mode: red, the 6th mode: brown; the 8th mode: blue; the 10th mode: green).

Then, as with the previous problem, we can use these POD-CSI models instead of the full DGTD formulations to compute the electromagnetic fields with the testing parameters $\theta^{1}=\{(5.1,3.4,2.1,1.4)\}, \theta^{2}=\{(5.4,3.4,2.3,1.3)\}$, and $\theta^{3}=\{(5.5,3.7,2.4,1.7)\}$ in order to assess the accuracy of the POD-CSI method. The 1-D x-wise distribution of the real part of $H_{y}$ in the Fourier domain over the last period of simulation along $y=0$ is displayed in Figure 10 (a),(c),(e). The bistatic RCS is shown in Figure 10 (b),(d),(f), matching well with those obtained by the DGTD method. The time evolution of the relative projection error $e_{\mathbf{u}, \text { Pro }}$, the POD-CSI error $e_{\mathbf{u}, \text { POD-CSI }}$ and the surrogate error $\widehat{e}_{\mathbf{u}, \text { POD-CSI }}(\mathbf{u} \in\{\mathbf{E}, \mathbf{H}\})$ for the testing parameters $\theta^{1}, \theta^{2}$, and $\theta^{3}$ are shown in Figure 11. The corresponding max errors are listed in Table 9.

The performance results obtained by the POD-CSI and DGTD methods with $\mathbb{P}_{2}$ approximation are summarized in Table 10. The CPU time of the DGTD method is $6.007 \times 10^{2} \mathrm{~s}$ and 


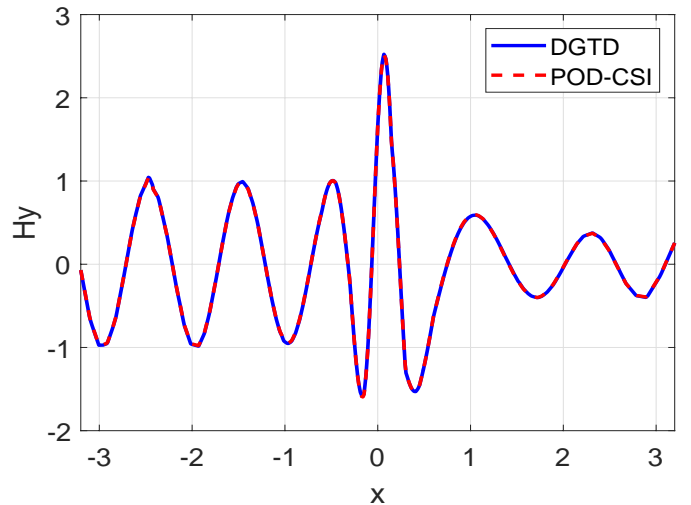

(a)

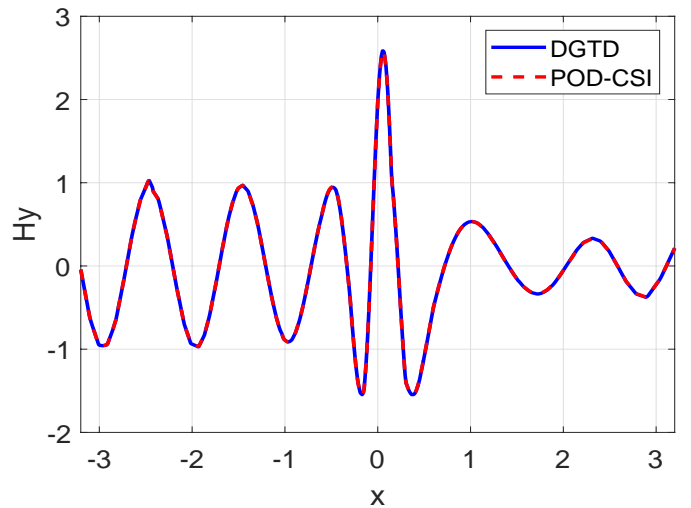

(c)

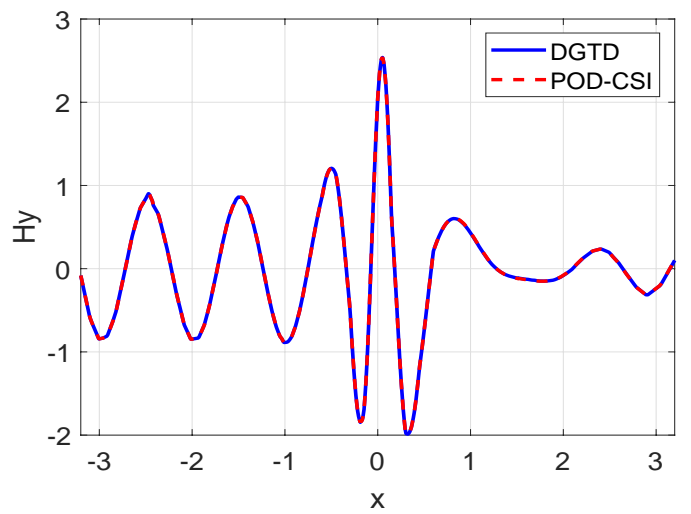

(e)

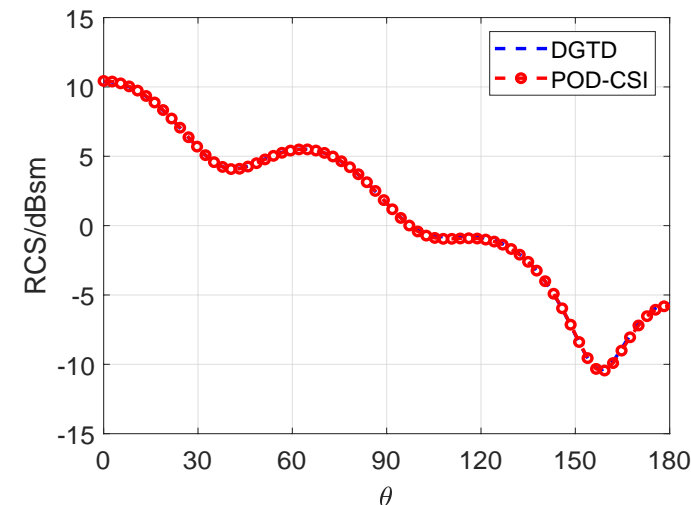

(b)

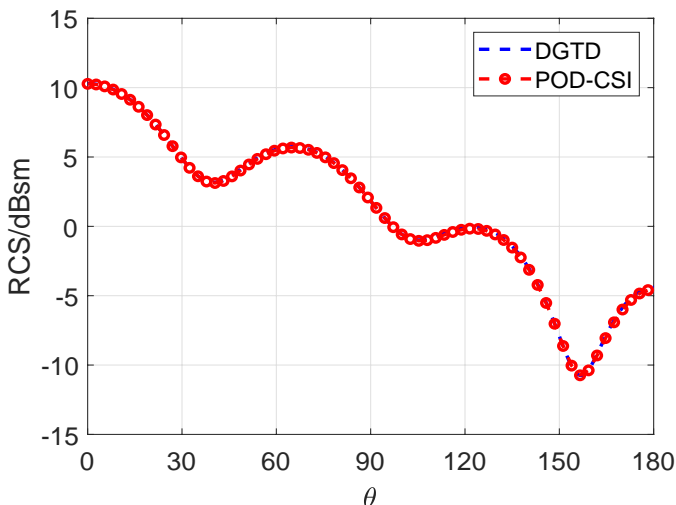

(d)

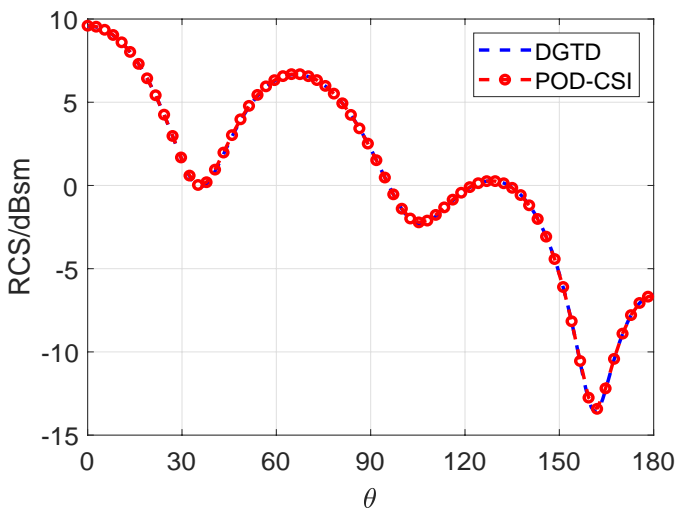

(f)

Figure 10: Scattering of plane wave by a multi-layer heterogeneous medium: 1-D plots of the real part of DGTD and POD-CSI solutions (DFT) of $H_{y}$ along $y=0$ (left), and bistatic RCS (right) based on the DGTD and PODCSI solutions with the testing parameters $\theta^{1}=\{(5.1,3.4,2.1,1.4)\}(\mathrm{a})-(\mathrm{b}), \theta^{2}=\{(5.4,3.4,2.3,1.3)\}$ (c)-(d), and $\theta^{3}=\{(5.5,3.7,2.4,1.7)\}(\mathrm{e})-(\mathrm{f})$.

Table 9: Scattering of plane wave by a dielectric cylinder: the max relative projection error $e_{\mathbf{u}, \text { Pro }}$, the max total relative error $e_{\mathbf{u}, \mathrm{POD}-\mathrm{CSI}}$, and the max surrogate error $\widehat{e}_{\mathbf{u}, \text { POD-CSI }}(\mathbf{u}=\mathbf{E}, \mathbf{H})$ for the testing parameters $\theta^{1}, \theta^{2}$, and $\theta^{3}$.

\begin{tabular}{ccccccc}
\hline Test parameters & $e_{\mathbf{E}, \text { Pro }}$ & $e_{\mathbf{E}, \text { POD-CSI }}$ & $\widehat{e}_{\mathbf{u}, \text { POD-CSI }}$ & $e_{\mathbf{H}, \text { Pro }}$ & $e_{\mathbf{H}, \text { POD-CSI }}$ & $\widehat{e}_{\mathbf{u}, \text { POD-CSI }}$ \\
\hline$\theta^{1}$ & $3.749 \times 10^{-3}$ & $5.880 \times 10^{-3}$ & $5.148 \times 10^{-3}$ & $4.432 \times 10^{-3}$ & $6.989 \times 10^{-3}$ & $6.805 \times 10^{-3}$ \\
$\theta^{2}$ & $3.685 \times 10^{-3}$ & $5.011 \times 10^{-3}$ & $4.742 \times 10^{-3}$ & $4.073 \times 10^{-3}$ & $5.802 \times 10^{-3}$ & $5.776 \times 10^{-3}$ \\
$\theta^{3}$ & $3.751 \times 10^{-3}$ & $4.413 \times 10^{-3}$ & $4.530 \times 10^{-3}$ & $4.408 \times 10^{-3}$ & $5.172 \times 10^{-3}$ & $4.611 \times 10^{-3}$ \\
\hline
\end{tabular}



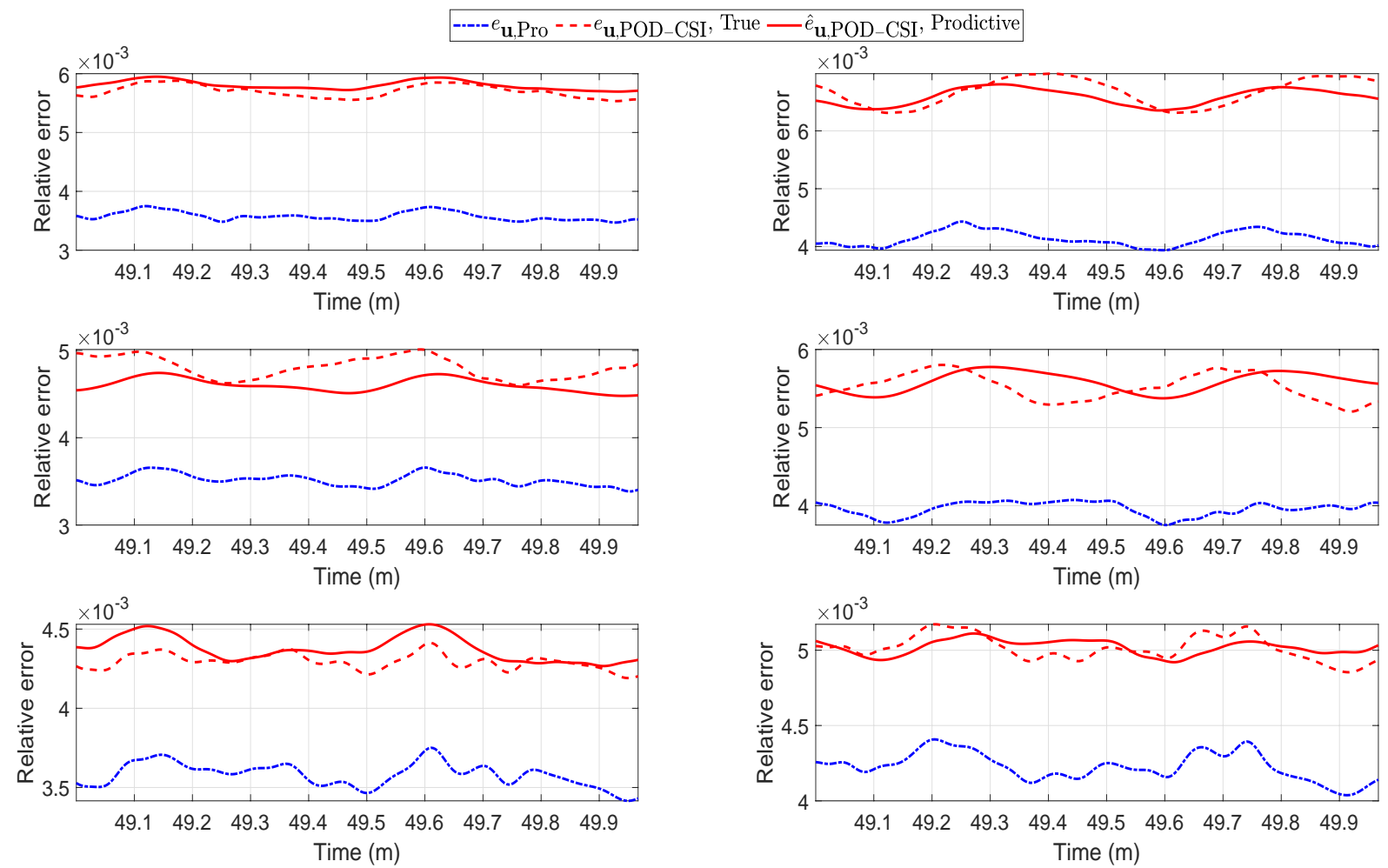

Figure 11: Scattering of plane wave by a multi-layer heterogeneous medium: the time evolution of the relative projection error $e_{\mathbf{u}, \text { Pro }}$, the relative POD-CSI error $e_{\mathbf{u}, \text { POD-CSI }}$, and the surrogate error $\widehat{e}_{\mathbf{u}, \text { POD-CSI }}$ for $\mathbf{E}$ (left) and $\mathbf{H}$ (right) with the testing parameters $\theta^{1}$ (top), $\theta^{2}$ (middle), and $\theta^{1}$ (bottom).

the online cost of the POD-CSI is $1.489 \mathrm{~s}$. This corresponds to a speed-up of 403, which show the significantly enhanced efficiency of the POD-CSI method.

Table 10: Scattering of plane wave by a multi-layer heterogeneous medium: the comparison between the POD-CSI (offline and online) and DGTD methods in terms of CPU time. The unit of time cost is second.

\begin{tabular}{ccccc}
\hline & Offline & \multicolumn{3}{c}{ Online } \\
\hline Snapshots & Two-step POD & CSI & POD-CSI & DGTD \\
$4.866 \times 10^{4}$ & $1.699 \times 10^{2}$ & $1.607 \times 10^{2}$ & 1.489 & $6.007 \times 10^{2}$ \\
\hline
\end{tabular}

\section{Conclusion}

In this paper, we have proposed a non-intrusive POD-CSI method for the parameterized electromagnetic scattering problems. Using the two-step POD method, the time- and parameterindependent POD basis functions are extracted from the collection of the full-order solutions (snapshots) generated by the DGTD method at some different parameter locations. Using the SVD method, the principal components of the reduced coefficient matrices of high fidelity solutions onto the RB subspace spanned by the POD basis functions are extracted. The discrete time- and parameter-modes for the reduced coefficients are approximated by using the CSI method. The offline stage includes the generation of snapshots, the construction of POD basis functions and the approximation of coefficient matrices based the CSI method, while the online stage only performs the recovery of the reduced order solutions based on the POD-CSI method. 
In particular, the offline and online stages of the POD-CSI method are completely decoupled. Besides, a surrogate error model is used to estimate the error of the POD-CSI method. Numerical results indicate that the POD-CSI is a promising method in electromagnetic simulations. Future research directions include more realistic 3-D simulations, the reduction of parameterized geometry and the improvement of the extrapolation accuracy of ROM.

\section{Acknowledgments}

The authors are very grateful for the constructive advices from anonymous reviewers and the help of the editors.

\section{Declarations}

\section{Fundings}

This research was supported by NSFC (Grant No. 61772003) and Key Projects of Applied Basic Research in Sichuan Province (Grant No. 2020YJ0216).

\section{Conflicts of interest/Competing interests}

We declare that we have no financial and personal relationships with other people or organizations that can inappropriately influence our work, there is no professional or other personal interest of any nature or kind in any product, service and/or company that could be construed as influencing the position presented in, or the review of, the manuscript entitled.

Availability of data and material

Not applicable

Code availability

Not applicable

\section{References}

[1] J. S. Hesthaven, G. Rozza, B. Stamm, et al., Certified reduced basis methods for parametrized partial differential equations, Springer, 2016.

[2] M. Guo, J. S. Hesthaven, Data-driven reduced order modeling for time-dependent problems, Computer Methods in Applied Mechanics and Engineering 345 (2019) 75-99.

[3] S. Georgaka, G. Stabile, G. Rozza, M. J. Bluck, Parametric POD-Galerkin model order reduction for unsteady-state heat transfer problems, Communications in Computational Physics 27 (1) (2020) 1-32.

[4] F. Vidal-Codina, N. C. Nguyen, J. Peraire, Computing parametrized solutions for plasmonic nanogap structures, Journal of Computational Physics 366 (2018) 89-106.

[5] K. Yee, Numerical solution of initial boundary value problems involving Maxwell's equations in isotropic media, IEEE Transactions on Antennas and Propagation 14 (3) (1966) 302-307.

[6] J. S. Hesthaven, T. Warburton, Nodal discontinuous Galerkin methods: algorithms, analysis, and applications, Springer Science \& Business Media, 2007. 
[7] P. Benner, S. Gugercin, K. Willcox, A survey of projection-based model reduction methods for parametric dynamical systems, SIAM Review 57 (4) (2015) 483-531.

[8] B. Peherstorfer, K. Willcox, M. Gunzburger, Survey of multifidelity methods in uncertainty propagation, inference, and optimization, SIAM Review 60 (3) (2018) 550-591.

[9] B. Haasdonk, M. Ohlberger, Efficient reduced models and a posteriori error estimation for parametrized dynamical systems by offline/online decomposition, Mathematical and Computer Modelling of Dynamical Systems 17 (2) (2011) 145-161.

[10] D. Pasetto, M. Putti, W. W.-G. Yeh, A reduced-order model for groundwater flow equation with random hydraulic conductivity: application to monte carlo methods, Water Resources Research 49 (6) (2013) 3215-3228.

[11] J. S. Hesthaven, B. Stamm, S. Zhang, Efficient greedy algorithms for high-dimensional parameter spaces with applications to empirical interpolation and reduced basis methods, ESAIM: Mathematical Modelling and Numerical Analysis 48 (1) (2014) 259-283.

[12] L. Sirovich, Turbulence and the dynamics of coherent structures. I. Coherent structures, Quarterly of Applied Mathematics 45 (3) (1987) 561-571.

[13] M. Rathinam, L. R. Petzold, A new look at proper orthogonal decomposition, SIAM Journal on Numerical Analysis 41 (5) (2003) 1893-1925.

[14] R. Pinnau, Model reduction via proper orthogonal decomposition, in: Model order reduction: theory, research aspects and applications, Springer, 2008, pp. 95-109.

[15] S. Chaturantabut, D. C. Sorensen, A state space error estimate for POD-DEIM nonlinear model reduction, SIAM Journal on Numerical Analysis 50 (1) (2012) 46-63.

[16] K. Li, T.-Z. Huang, L. Li, S. Lanteri, POD-based model order reduction with an adaptive snapshot selection for a discontinuous Galerkin approximation of the time-domain Maxwell's equations, Journal of Computational Physics 396 (2019) 106-128.

[17] F. Ballarin, A. Manzoni, A. Quarteroni, G. Rozza, Supremizer stabilization of PODGalerkin approximation of parametrized steady incompressible Navier-Stokes equations, International Journal for Numerical Methods in Engineering 102 (5) (2015) 1136-1161.

[18] Q. Wang, J. S. Hesthaven, D. Ray, Non-intrusive reduced order modeling of unsteady flows using artificial neural networks with application to a combustion problem, Journal of Computational Physics 384 (2019) 289-307.

[19] O. Lass, S. Volkwein, POD-Galerkin schemes for nonlinear elliptic-parabolic systems, SIAM Journal on Scientific Computing 35 (3) (2013) A1271-A1298.

[20] S. Ullmann, M. Rotkvic, J. Lang, POD-Galerkin reduced-order modeling with adaptive finite element snapshots, Journal of Computational Physics 325 (2016) 244-258.

[21] F. Ballarin, E. Faggiano, S. Ippolito, A. Manzoni, A. Quarteroni, G. Rozza, R. Scrofani, Fast simulations of patient-specific haemodynamics of coronary artery bypass grafts based on a POD-Galerkin method and a vascular shape parametrization, Journal of Computational Physics 315 (2016) 609-628. 
[22] M. Strazzullo, F. Ballarin, G. Rozza, POD-Galerkin model order reduction for parametrized nonlinear time dependent optimal flow control: an application to shallow water equations, Journal of Scientific Computing 83 (2020). https://doi.org/10.1007/s10915-020-01232-x.

[23] U. Baur, P. Benner, L. Feng, Model order reduction for linear and nonlinear systems: a system-theoretic perspective, Archives of Computational Methods in Engineering 21 (4) (2014) 331-358.

[24] K. Carlberg, M. Barone, H. Antil, Galerkin v. least-squares Petrov-Galerkin projection in nonlinear model reduction, Journal of Computational Physics 330 (2017) 693-734.

[25] H. V. Ly, H. T. Tran, Modeling and control of physical processes using proper orthogonal decomposition, Mathematical and Computer Modelling 33 (1-3) (2001) 223-236.

[26] K. Li, T.-Z. Huang, L. Li, S. Lanteri, L. Xu, B. Li, A reduced-order discontinuous Galerkin method based on POD for electromagnetic simulation, IEEE Transactions on Antennas and Propagation 66 (1) (2017) 242-254.

[27] H. Fu, H. Wang, Z. Wang, POD/DEIM reduced-order modeling of time-fractional partial differential equations with applications in parameter identification, Journal of Scientific Computing 74 (1) (2018) 220-243.

[28] Z. Luo, H. Ren, A reduced-order extrapolated finite difference iterative method for the riemann-liouville tempered fractional derivative equation, Applied Numerical Mathematics 157 (2020) 307-314.

[29] J. Shen, J. R. Singler, Y. Zhang, HDG-POD reduced order model of the heat equation, Jounral of Computational and Applied Mathematics 362 (2019) 663-679.

[30] S. Yıldız, P. Goyal, P. Benner, B. Karasozen, Data-driven learning of reduced-order dynamics for a parametrized shallow water equation, 2020. arxiv preprint arxiv:2007.14079.

[31] J. Yu, C. Yan, Z. Jiang, W. Yuan, S. Chen, Adaptive non-intrusive reduced order modeling for compressible flows, Journal of Computational Physics 397 (2019) 108855.

[32] J. Yu, C. Yan, M. Guo, Non-intrusive reduced-order modeling for fluid problems: a brief review, Proceedings of the Institution of Mechanical Engineers, Part G: Journal of Aerospace Engineering 233 (16) (2019) 5896-5912.

[33] F. Casenave, A. Ern, T. Leliévre, A nonintrusive reduced basis method applied to aeroacoustic simulations, Advances in Computational Mathematics 41 (5) (2015) 961-986.

[34] J. S. Hesthaven, S. Ubbiali, Non-intrusive reduced order modeling of nonlinear problems using neural networks, Journal of Computational Physics 363 (2018) 55-78.

[35] O. San, R. Maulik, M. Ahmed, An artificial neural network framework for reduced order modeling of transient flows, Communications in Nonlinear Science and Numerical Simulation 77 (2019) 271-287.

[36] M. Guo, J. S. Hesthaven, Reduced order modeling for nonlinear structural analysis using gaussian process regression, Computer Methods in Applied Mechanics and Engineering 341 (2018) 807-826. 
[37] C. Audouze, F. De Vuyst, P. B. Nair, Nonintrusive reduced-order modeling of parametrized time-dependent partial differential equations, Numerical Methods for Partial Differential Equations 29 (5) (2013) 1587-1628.

[38] D. Xiao, F. Fang, C. C. Pain, I. M. Navon, P. Salinas, Non-intrusive reduced order modeling of multi-phase flow in porous media using the POD-RBF method, Journal of Computational Physics (2015).

[39] M. Dehghan, M. Abbaszadeh, The use of proper orthogonal decomposition (POD) meshless RBF-FD technique to simulate the shallow water equations, Journal of Computational Physics 351 (2017) 478-510.

[40] P. G. Constantine, D. F. Gleich, Y. Hou, J. Templeton, Model reduction with mapreduceenabled tall and skinny singular value decomposition, SIAM Journal on Scientific Computing 36 (5) (2014) S166-S191.

[41] X. Sun, X. Pan, J.-I. Choi, A non-intrusive reduced-order modeling method using polynomial chaos expansion, 2019. arxiv preprint arxiv:1903.10202.

[42] T. Bui-Thanh, M. Damodaran, K. Willcox, Proper orthogonal decomposition extensions for parametric applications in compressible aerodynamics, in: Proceedings of the 21st Applied Aerodynamics AIAA Conference, Orlando, Florida, 2003.

[43] M. Oulghelou, C. Allery, Non intrusive method for parametric model order reduction using a bi-calibrated interpolation on the grassmann manifold, Journal of Computational Physics (2020) 109924.

[44] E. Schmidt, On the theory of linear and nonlinear integral equations. I. development of arbitrary function according to systems prescribed, Mathematical Annals 63 (1907) 433476 .

[45] C. Eckart, G. Young, The approximation of one matrix by another of lower rank, Psychometrika 1 (3) (1936) 211-218.

[46] K. Kunisch, S. Volkwein, Galerkin proper orthogonal decomposition methods for a general equation in fluid dynamics, SIAM Journal on Numerical Analysis 40 (2) (2002) 492-515.

[47] S. Georgaka, G. Stabile, K. Star, G. Rozza, M. J. Bluck, A hybrid reduced order method for modelling turbulent heat transfer problems, Computers \& Fluids (2020) 104615.

[48] S. McKinley, M. Levine, Cubic spline interpolation, College of the Redwoods 45 (1) (1998) 1049-1060.

[49] G. H. Behforooz, A comparison of the E(3) and not-a-knot cubic splines, Applied Mathematics and Computation 72 (2-3) (1995) 219-223.

[50] M. S. Hasan, S. K. Islam, B. J. Blalock, Modeling of soi four-gate transistor (g4fet) using multidimensional spline interpolation method, Microelectronics Journal 76 (2018) 33-42.

[51] M. S. Hasan, S. Amer, S. K. Islam, G. S. Rose, Multivariate cubic spline: a versatile DC modeling technique suitable for different deep submicron transistors, in: Proceedings of IEEE SoutheastCon, 2019, pp. 1-8.

[52] R.-H. Wang, Multivariate spline functions and their applications, Springer Science \& Business Media, 2013. 
[53] L. D. Avendaño-Valencia, E. N. Chatzi, K. Y. Koo, J. M. Brownjohn, Gaussian process time-series models for structures under operational variability, Frontiers in Built Environment 3 (2017) 69 .

[54] S. Trehan, K. T. Carlberg, L. J. Durlofsky, Error modeling for surrogates of dynamical systems using machine learning, International Journal for Numerical Methods in Engineering 112 (12) (2017) 1801-1827.

[55] M. El Bouajaji, V. Dolean, M. J. Gander, S. Lanteri, R. Perrussel, Discontinuous Galerkin discretizations of optimized Schwarz methods for solving the time-harmonic Maxwell equations, Electronic Transactions on Numerical Analysis 44 (2015) 572-592. 